JOURNAL OF

SYMPLECTIC GEOMETRY

Volume 3, Number 4, 671-701, 2005

\title{
THREE APPROACHES TOWARDS FLOER HOMOLOGY OF COTANGENT BUNDLES
}

\author{
JOA WEBER
}

Consider the cotangent bundle of a closed Riemannian manifold and an almost complex structure close to the one induced by the Riemannian metric. For Hamiltonians which grow, for instance, quadratically in the fibers outside a compact set, one can define Floer homology and show that it is naturally isomorphic to singular homology of the free loop space. We review the three isomorphisms constructed by Viterbo [16], Salamon-Weber [18] and Abbondandolo-Schwarz [14]. The theory is illustrated by calculating Morse and Floer homology in case of the Euclidean $n$-torus. Applications include existence of noncontractible periodic orbits of compactly supported Hamiltonians on open unit disc cotangent bundles which are sufficiently large over the zero section.

\section{Chain group and boundary operators}

Let $M$ be a closed smooth manifold and fix a Riemannian metric. Let $\nabla$ be the associated Levi-Civita connection. This endows the free loop space $\mathcal{L} M=C^{\infty}\left(S^{1}, M\right)$ with an $L^{2}$ and a $W^{1,2}$ metric given by

$$
\langle\xi, \eta\rangle_{L^{2}}=\int_{0}^{1}\langle\xi(t), \eta(t)\rangle d t, \quad\langle\xi, \eta\rangle_{W^{1,2}}=\langle\xi, \eta\rangle_{L^{2}}+\left\langle\nabla_{t} \xi, \nabla_{t} \eta\right\rangle_{L^{2}},
$$

where $\xi$ and $\eta$ are smooth vector fields along $x \in \mathcal{L} M$. Here and throughout, we identify $S^{1}=\mathbb{R} / \mathbb{Z}$ and think of $x \in \mathcal{L} M$ as a smooth map $x$ : $\mathbb{R} \rightarrow M$ which satisfies $x(t+1)=x(t)$. Fix a time-dependent function $V \in C^{\infty}\left(S^{1} \times M\right)$ and set $V_{t}(q):=V(t, q)$. The classical action functional on $\mathcal{L} M$ is defined by

$$
\mathcal{S}_{V}(x):=\int_{0}^{1}\left(\frac{1}{2}|\dot{x}(t)|^{2}-V_{t}(x(t))\right) d t .
$$


The set $\mathcal{P}(V)$ of critical points consists of the 1-periodic solutions of the second-order ordinary differential equation (ODE)

$$
\nabla_{t} \partial_{t} x=-\nabla V_{t}(x) .
$$

Here $\nabla V_{t}$ denotes the gradient vector field. These solutions are called perturbed closed geodesics. Two features make the functional $\mathcal{S}_{V}$ accessible to standard variational methods, boundedness from below and finiteness of the Morse index ${ }^{1} \operatorname{ind}_{V}(x)$ of every critical point $x$. A critical point is called nondegenerate if its Hessian is nondegenerate. A function with nondegenerate critical points only is a Morse function. If $\mathcal{S}_{V}$ is Morse, the change of topology of the sublevel set

$$
\left\{\mathcal{S}_{V} \leq a\right\}:=\left\{x \in \mathcal{L} M \mid \mathcal{S}_{V}(x) \leq a\right\},
$$

when $a \in \mathbb{R}$ passes through a critical value is the subject of classical Morse theory leading to a CW-complex homotopy equivalent to $\left\{\mathcal{S}_{V} \leq a\right\}$ (see, e.g. [1]). In the case $V=0$, we use the notation $\mathcal{L}^{a} M:=\left\{\mathcal{S}_{0} \leq a\right\}$.

$W^{1,2}$ Morse homology. A geometric reincarnation of the idea of encoding the topology of a sublevel set in terms of a Morse function came (back) to light in 1982 through the work of Witten [2]. Roughly speaking, the MorseWitten complex consists of chain groups generated by the critical points of a Morse function and a boundary operator which counts flow lines of the negative gradient flow between critical points of Morse index difference one (for details see, e.g. [3] and [4]). In recent years, Abbondandolo and Majer [5] extended the theory from finite dimensions to a class of Hilbert manifolds. The free loop space fits into this framework after completion with respect to the Sobolev $W^{1,2}$ norm. From now on, we assume that $\mathcal{S}_{V}$ is a Morse function. (A proof that this holds for a generic potential $V$ is given in $[\mathbf{6}]$.) Then the set

$$
\mathcal{P}^{a}(V):=\left\{x \in \mathcal{P}(V) \mid \mathcal{S}_{V}(x) \leq a\right\}
$$

is finite for every real number $a$. Also from now on, we assume that $a$ is a regular value of $\mathcal{S}_{V}$. The chain groups are the free abelian groups generated by $\mathcal{P}^{a}(V)$ and graded by the Morse index, namely

$$
C_{k}^{a}(V)=\bigoplus_{\substack{x \in \mathcal{P}^{a}(V) \\ \operatorname{ind}_{V}(x)=k}} \mathbb{Z} x, \quad k \in \mathbb{Z} .
$$

Our convention is that the direct sum over an empty set equals $\{0\}$. The negative of the $W^{1,2}$ gradient vector field induces a flow on the $\left(W^{1,2}\right.$ completion of the) loop space whose unstable manifolds are of finite dimension and whose stable manifolds are of finite codimension. Let us choose an orientation of the unstable manifold of every critical point. If the Morse-Smale

\footnotetext{
${ }^{1}$ The dimension of the largest subspace on which the Hessian is negative definite.
} 
condition holds (this means that all stable and unstable manifolds intersect transversally), then there are only finitely many flow lines between critical points of index difference one. These are called isolated flow lines. Each one inherits an orientation, because it is the intersection of an oriented and a cooriented submanifold. Let the characteristic sign of an isolated flow line be +1 if the inherited orientation coincides with the one provided by the flow and -1 else. Counting isolated flow lines with characteristic signs defines the Morse-Witten boundary operator. The associated homology groups $\operatorname{HM}_{*}^{a}\left(\mathcal{L} M, \mathcal{S}_{V}, W^{1,2}\right)$ are called $W^{1,2}$ Morse homology. By the theory of Abbondandolo and Majer, it is naturally isomorphic to integral singular homology $\mathrm{H}_{*}\left(\left\{\mathcal{S}_{V} \leq a\right\}\right)$. If $\mathcal{L} M$ has several connected components $\mathcal{L}_{\alpha} M$, then there is a separate isomorphism for each of them. The label $\alpha$ denotes a homotopy class of free loops in $M$.

$\boldsymbol{L}^{\mathbf{2}}$ Morse homology. Replacing the $W^{1,2}$ metric on the free loop space by the $L^{2}$ metric leads to a new boundary operator on the chain groups (2), namely by counting negative $L^{2}$ gradient "flow lines." In fact the $L^{2}$ metric gives rise only to a semiflow in forward time and so we view-in the spirit of Floer theory - the negative gradient flow equation on the loop space as a partial differential equation (PDE) for smooth cylinders in $M$. Flow lines are then replaced by solutions $u: \mathbb{R} \times S^{1} \rightarrow M$ of the heat equation

$$
\partial_{s} u-\nabla_{t} \partial_{t} u-\nabla V_{t}(u)=0
$$

which satisfy

$$
\lim _{s \rightarrow \pm \infty} u(s, t)=x^{ \pm}(t), \quad \lim _{s \rightarrow \pm \infty} \partial_{s} u(s, t)=0 .
$$

Here $x^{ \pm} \in \mathcal{P}(V)$ and the limits are supposed to be uniform in $t$. Stable manifolds can still be defined via the forward semiflow, whereas to define unstable manifolds we use the heat flow lines (3). The former are of finite codimension and the latter are of finite dimension. Hence characteristic signs can be assigned to isolated (index difference 1) flow lines just as in the case of $W^{1,2}$ Morse homology above. The parabolic moduli space $\mathcal{M}^{0}\left(x^{-}, x^{+} ; V\right)$ is the set of solutions of equations (3) and (4). In this setting, we say that the Morse-Smale condition holds, if the linear operator obtained by linearizing equation (3) at a solution $u$ is onto for all $u \in \mathcal{M}^{0}\left(x^{-}, x^{+} ; V\right)$ and for all $x^{ \pm} \in \mathcal{P}(V)$. In this case, the moduli space $\mathcal{M}^{0}\left(x^{-}, x^{+} ; V\right)$ is a smooth manifold of dimension ind ${ }_{V}\left(x^{-}\right)-$ind $_{V}\left(x^{+}\right)$. In the case of index difference 1 , the quotient by the free time shift action is a finite set. Counting its elements with characteristic signs defines the $L^{2}$ Morse-Witten boundary operator $\partial^{M}$. The associated homology $\operatorname{HM}_{*}^{a}\left(\mathcal{L} M, \mathcal{S}_{V}, L^{2}\right)$ is called $L^{2}$ Morse homology. It is naturally isomorphic to $\mathrm{H}_{*}\left(\left\{\mathcal{S}_{V} \leq a\right\}\right)$. (We should emphasize that this is work in progress [7].) 
Floer homology. The critical points of $\mathcal{S}_{V}$ can be interpreted via the Legendre transformation as the critical points of the symplectic action functional on the free loop space of $T^{*} M$ given by

$$
\mathcal{A}_{V}(z)=\mathcal{A}_{H_{V}}(z):=\int_{0}^{1}\left(\left\langle y(t), \partial_{t} x(t)\right\rangle-H_{V}(t, x(t), y(t))\right) d t .
$$

Here $z=(x, y)$ where $x: S^{1} \rightarrow M$ is a smooth map and $y(t) \in T_{x(t)}^{*} M$ depends smoothly on $t \in S^{1}$. The Hamiltonian $H_{V}: S^{1} \times T^{*} M \rightarrow \mathbb{R}$ is given by

$$
H_{V}(t, q, p)=\frac{1}{2}|p|^{2}+V_{t}(q)
$$

A loop $z=(x, y)$ in $T^{*} M$ is a critical point of $\mathcal{A}_{V}$ iff $x$ is a critical point of $\mathcal{S}_{V}$ and $y(t) \in T_{x(t)}^{*} M$ is related to $\partial_{t} x(t) \in T_{x(t)} M$ via the isomorphism $g: T M \rightarrow T^{*} M$ induced by the Riemannian metric. For such loops $z$, the symplectic action $\mathcal{A}_{V}(z)$ agrees with the classical action $\mathcal{S}_{V}(x)$.

In contrast to the classical action, the symplectic action is in general neither bounded below nor do the critical points admit finite Morse indices, and most importantly its $L^{2}$ gradient does not define a flow on the loop space. It was a great achievement of Floer [8] to nevertheless set up a MorseWitten-type complex. His key idea was to reinterpret the negative $L^{2}$ gradient equation as elliptic PDE for maps from the cylinder to the symplectic manifold imposing appropriate boundary conditions to make the problem Fredholm. Floer's original setup was a closed symplectic manifold subject to two topological assumptions to ensure compactness of moduli spaces and existence of a natural grading. A Hamiltonian function $H$ and an almost complex structure $J$ need to be chosen to define the Floer complex. The power of Floer theory lies in the fact that Floer homology is independent of these choices. This is called the Floer continuation principle. Floer showed that if $H$ is autonomous and a $C^{2}$-small Morse function, then the Floer chain complex equals the Morse-Witten complex. Hence Floer homology is naturally isomorphic to singular integral homology of the closed symplectic manifold itself. For introductory reading, we refer to Salamon's lecture notes $[\mathbf{9}]$ and the recent survey by Laudenbach $[\mathbf{1 0}]$. A discussion on a more advanced level, also including applications of Floer theory, can be found in Chapter 12 of [11].

Now consider the cotangent bundle $T^{*} M$ equipped with its canonical symplectic structure $\omega_{0}=-d \theta$, where $\theta$ is the Liouville form. Since the symplectic form is exact and the first Chern class of $T^{*} M$ with respect to the metric-induced almost complex structure vanishes, both topological assumptions of Floer are met. The former excludes existence of nonconstant $J$-holomorphic spheres, which is an obstruction towards compactness of the moduli spaces, and the latter implies that the Conley-Zehnder index of 1-periodic Hamiltonian orbits is well defined. Since the 1-periodic orbits 
of the Hamiltonian flow are precisely the critical points of $\mathcal{A}_{V}$ and these coincide with the critical points of $\mathcal{S}_{V}$ up to natural identification, the Floer chain groups are again given by the free abelian groups (2). (The standard Floer grading is the negative Conley-Zehnder index, which is proved in [6] to equal the Morse index, up to a constant if $M$ is not orientable.) The Riemannian metric on $M$ provides the isomorphism

$$
T_{(x, y)} T^{*} M \longrightarrow T_{x} M \oplus T_{x}^{*} M,
$$

which takes the derivative of a curve $\mathbb{R} \rightarrow T^{*} M: t \mapsto z(t)=(x(t), y(t))$ to the derivatives of the two components, namely

$$
\partial_{t} z(t) \longmapsto\left(\partial_{t} x(t), \nabla_{t} y(t)\right) .
$$

The metric also induces an almost complex structure $J_{g}$ and a metric $G_{g}$ on $T^{*} M$. These and the symplectic form are represented by

$$
J_{g}=\left(\begin{array}{cc}
0 & -g^{-1} \\
g & 0
\end{array}\right), \quad G_{g}=\left(\begin{array}{cc}
g & 0 \\
0 & g^{-1}
\end{array}\right), \quad \omega_{0}=\left(\begin{array}{cc}
0 & -1 \\
1 & 0
\end{array}\right) .
$$

These three structures are compatible in the sense that $\omega_{0}\left(\cdot, J_{g} \cdot\right)=G_{g}(\cdot, \cdot)$. Flow lines are then replaced by solutions $w: \mathbb{R} \times S^{1} \rightarrow T^{*} M$ of Floer's equation

$$
\partial_{s} w+J_{g}(w) \partial_{t} w-\nabla H_{V}(t, w)=0
$$

It is the negative $L^{2}$ gradient equation for the symplectic action viewed as an elliptic PDE. Its solutions are called Floer trajectories or Floer cylinders. If we identify $T^{*} M$ and $T M$ via the metric isomorphism and represent Floer's equation with respect to the splitting (5), we obtain the pair of equations

$$
\partial_{s} u-\nabla_{t} v-\nabla V_{t}(u)=0, \quad \nabla_{s} v+\partial_{t} u-v=0
$$

for $(u, v): \mathbb{R} \times S^{1} \rightarrow T M$. The Floer moduli space $\mathcal{M}^{1}\left(x^{-}, x^{+} ; V\right)$ is the set of solutions of equation (6) subject to the boundary conditions

$$
\lim _{s \rightarrow \pm \infty} u(s, t)=x^{ \pm}(t), \quad \lim _{s \rightarrow \pm \infty} v(s, t)=\partial_{t} x^{ \pm}(t),
$$

and $\partial_{s} u$ and $\nabla_{s} v$ converge to zero as $|s| \rightarrow \infty$, and all limits are uniformly in $t$. If the Morse-Smale condition is satisfied, then $\mathcal{M}^{1}\left(x^{-}, x^{+} ; V\right)$ is a smooth manifold whose dimension is given by the difference of Morse indices. Since $T^{*} M$ is noncompact, we do not obtain for free uniform a priori $C^{0}$-bounds for the Floer solutions as in the standard case of a closed symplectic manifold. Such bounds were established in 1992 by Cieliebak in his diploma thesis $[\mathbf{1 2}]$ and recently extended to a class of radial Hamiltonians by the author $[\mathbf{1 3}]$ and to another class of not necessarily radial Hamiltonians by Abbondandolo and Schwarz [14]. Given these bounds, proving compactness and setting up Floer homology is standard. In the case that $\mathcal{L} T^{*} M$ has several connected components, Floer homology is defined for each of them separately and denoted by $\mathrm{HF}_{*}^{a}\left(T^{*} M, H_{V}, J_{g} ; \alpha\right)$. Here $\alpha$ denotes a homotopy class of free loops in $M$. (Throughout we identify homotopy classes of 
free loops in $M$ and in $T^{*} M$.) In fact Floer homology can be defined for classes of Hamiltonians more general than $H_{V}$; for instance, those growing quadratically in $p$ outside a compact set (see Section 2) or convex radial Hamiltonians (see Section 6).

Isomorphisms between the theories. When Floer homology for physical Hamiltonians of the type kinetic plus potential energy could be defined by 1992 due to Cieliebak's breakthrough, the obvious question was "What is it equal to?".

The first answer ${ }^{2}$ in the literature is due to Viterbo who conjectured in his 1994 ICM talk [15] - based on formal interpretation of the symplectic action functional as a generating function - that Floer homology of the cotangent bundle represents singular homology of the free loop space. In his 1996 preprint [16], he gave a beautiful line of argument (for the component $\mathcal{L}_{0} M$ of contractible loops, case $V=0$, coefficients in $\mathbb{Z}_{2}$ ). The idea is to view the time-1-map $\varphi_{1}$ of the Hamiltonian flow as an $r$-fold composition of symplectomorphisms close to the identity $\left(\right.$ set $\left.\psi:=\varphi_{1 / r}\right)$ in order to arrive at the well-known finite-dimensional approximation of the free loop space via broken geodesics. While the argument consists of numerous steps and the idea of each one is described in detail, not all technical details are provided.

Also in 1996, a first example was computed by Weber [17], namely Floer homology of the cotangent bundle of the Euclidean torus $\mathbb{T}^{n}=\mathbb{R}^{n} / 2 \pi \mathbb{Z}^{n}$ confirming the conjecture for all connected components of $\mathcal{L} \mathbb{T}^{n}$, for every $n \in \mathbb{N}$. This is reviewed in Section 5 .

In 2003, Salamon and Weber [18] proved existence of a natural isomorphism

$$
\mathrm{HM}_{*}^{a}\left(\mathcal{L} M, \mathcal{S}_{V}, L^{2}\right) \longrightarrow \mathrm{HF}_{*}^{a}\left(T^{*} M, H_{V}, J_{g}\right) .
$$

(Partial results were established in 1999 in the Ph.D. thesis [19].) The idea is to introduce a real parameter $\varepsilon>0$ and to replace the standard almost complex structure $J_{g}$ by $J_{\varepsilon^{-1} g}$. Both Floer homologies are naturally isomorphic by Floer continuation. The key step is then to prove that for every sufficiently small $\varepsilon$, the parabolic and elliptic moduli spaces can be identified. Hence the $\varepsilon$-Floer and the $L^{2}$ Morse chain complexes are identical.

In 2004, Abbondandolo and Schwarz [14] proved existence of a natural isomorphism

$$
\mathrm{HM}_{*}^{a}\left(\mathcal{L} M, \mathcal{S}_{V}, W^{1,2}\right) \longrightarrow \mathrm{HF}_{*}^{a}\left(T^{*} M, H_{V}, J_{g}\right)
$$

\footnotetext{
${ }^{2}$ By then Theorem 1.1 had been expected to be true by part of the community. For instance, the problem was proposed as a Ph.D. project to the author by Helmut Hofer during winter term 1993/1994 at ETH Zürich. In summer 1996 upon meeting Dietmar Salamon in Oberwolfach, we matched up and started our joint approach. A short time later [16] appeared. In private communication at a Warwick conference, around 1998, Matthias Schwarz first told me about an alternative approach via a mixed boundary value problem.
} 
by constructing a chain isomorphism in the case of orientable $M$. Their approach works for more general Hamiltonians and almost complex structures (see Section 2). The idea is to study a mixed boundary value problem for Floer half cylinders $w=(u, v):[0, \infty) \rightarrow T^{*} M$. For $s \rightarrow+\infty$, the standard Floer boundary condition (7) is imposed, whereas at $s=0$ the base loop $u(0, \cdot)$ is required to belong to an unstable manifold of the negative $W^{1,2}$ gradient flow of $\mathcal{S}_{V}$.

In 2004, Weber [13] extended the definition and computation of Floer homology to yet another class of Hamiltonians: convex radial ones (those of the form $h=h(|p|)$ with $\left.h^{\prime \prime} \geq 0\right)$.

The main result of Viterbo [16], Salamon and Weber [18], and Abbondandolo and Schwarz [14] as formulated in [18] is the following theorem.

Theorem 1.1. Let $M$ be a closed Riemannian manifold. Assume $\mathcal{S}_{V}$ is Morse and $a$ is either a regular value of $\mathcal{S}_{V}$ or is equal to infinity. Then there is a natural isomorphism

$$
\mathrm{HF}_{*}^{a}\left(T^{*} M, H_{V}, J_{g} ; R\right) \simeq \mathrm{H}_{*}\left(\left\{\mathcal{S}_{\mathcal{V}} \leq a\right\} ; R\right)
$$

for every principal ideal domain $R$. If $M$ is not simply connected, then there is a separate isomorphism for each component of the loop space. For a $<$ $b$, the isomorphism commutes with the inclusion induced homomorphisms $\mathrm{HF}_{*}^{a} \rightarrow \mathrm{HF}_{*}^{b}$ and $\mathrm{H}_{*}\left(\left\{\mathcal{S}_{\mathcal{V}} \leq a\right\}\right) \rightarrow \mathrm{H}_{*}\left(\left\{\mathcal{S}_{\mathcal{V}} \leq b\right\}\right)$.

We summarize the discussion by the diagram below in which arrows represent isomorphisms. The homologies are defined as usual by first perturbing to achieve Morse-Smale transversality and then taking the homology of the perturbed chain complex. The branch on the right-hand side indicates Viterbo's finite-dimensional approximation argument (which he actually formulated in terms of cohomology; see Section 4).

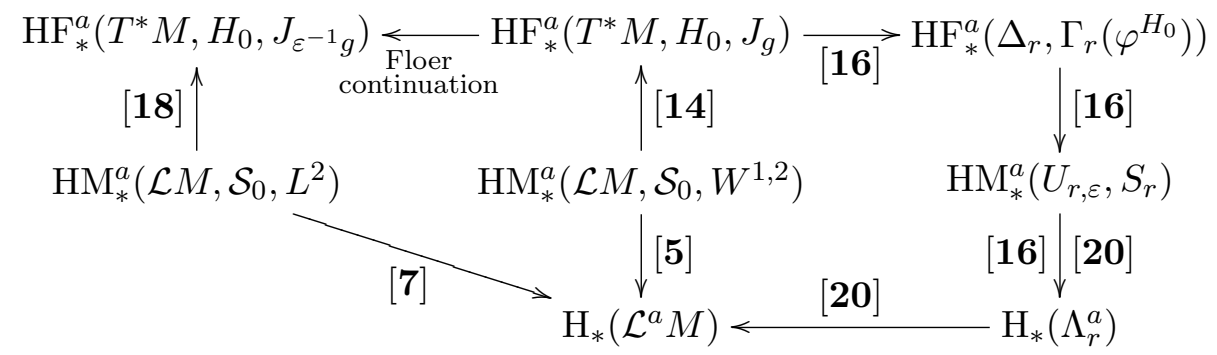

It would be interesting to fill in the missing link between $L^{2}$ and $W^{1,2}$ Morse homology by constructing a natural isomorphism.

The remaining part of this text is organized as follows. We present the three approaches towards Theorem 1.1 in chronologically reverse order in Sections 2-4. This way complexity increases - as it should be. In Section 5, 
we calculate Floer homology of the cotangent bundle of the Euclidean $n$ torus. An application of Theorem 1.1 to existence of noncontractible periodic orbits is reviewed in Section 6. For an application towards Arnold's chord conjecture, we refer to Cieliebak's paper [21].

\section{Mixed boundary value problem}

In three steps, we review the approach of Abbondandolo and Schwarz [14]. They assume for simplicity that $M$ is orientable.

First of all, the authors set up Floer theory for a more general class of Hamiltonians $H$ and almost complex structures $J$. Let $\mathcal{P}(H)$ denote the set of critical points of the symplectic action $\mathcal{A}_{H}$. These are precisely the 1-periodic orbits of the Hamiltonian flow on $T^{*} M$. The crucial (metricindependent) assumptions on $H$ are the following. Outside a compact set, $H$ is supposed to satisfy

$$
\begin{gathered}
d H(t, q, p) p \partial_{p}-H(t, q, p) \geq c_{0}|p|^{2}-c_{1}, \\
\left|\nabla_{q} H(t, q, p)\right| \leq c_{2}\left(1+|p|^{2}\right), \quad\left|\nabla_{p} H(t, q, p)\right| \leq c_{2}(1+|p|),
\end{gathered}
$$

for some constants $c_{0}>0$ and $c_{1}, c_{2} \geq 0$. Assumptions (H1) and (H2) guarantee that the set $\mathcal{P}^{a}(H)$ is finite for every real number $a$, whenever $\mathcal{A}_{H}$ is Morse (which we shall assume from now on, since it is true for generic $H$ ). More importantly, assumptions (H1) and (H2) allow the authors to establish $C^{0}$-bounds for Floer solutions associated to almost complex structures $J$ sufficiently $C^{\infty}$-close to $J_{g}$. Then the definition of the chain complex $\left(\mathrm{CF}_{*}(H), \partial_{*}(H, J)\right)$ proceeds by standard arguments. Any two choices of $J$ lead to isomorphic chain complexes. In contrast, homology is independent of $(H, J)$ and denoted by $\mathrm{HF}_{*}\left(T^{*} M\right)$.

Secondly, the Morse-Witten complex is defined for the Hilbert manifold $W^{1,2}\left(S^{1}, M\right)$ and the classical action $\mathcal{S}_{L}(x):=\int_{0}^{1} L(t, x(t), \dot{x}(t)) d t$. Here the admissible Lagrangians $L$ are those for which there exist constants $d_{0}>0$ and $d_{1} \geq 0$ such that

$$
\begin{gathered}
\nabla_{v v} L(t, q, v) \geq d_{0} \mathbf{1}, \\
\left|\nabla_{q q} L(t, q, v)\right| \leq d_{1}\left(1+|v|^{2}\right), \quad\left|\nabla_{q v} L(t, q, v)\right| \leq d_{1}(1+|v|), \\
\left|\nabla_{v v} L(t, q, v)\right| \leq d_{1},
\end{gathered}
$$

for all $(t, q, v) \in S^{1} \times T M$. Perturbing $L$ if necessary we assume from now on that $\mathcal{S}_{L}$ is Morse and denote the set of its critical points by $\mathcal{P}(L)$. The classical action exhibits a number of rather useful features: it satisfies the Palais-Smale condition, it is bounded from below and its critical points have finite Morse indices (equal to minus the corresponding Conley-Zehnder indices). ${ }^{3} \quad$ Choosing an auxiliary Morse-Smale metric $\mathcal{G}$ on the Hilbert

\footnotetext{
${ }^{3}$ The sign difference between $[\mathbf{6}, \mathbf{1 8}]$ and $[\mathbf{1 4}]$ is due to the different normalizations $\mu_{C Z}\left(t \mapsto e^{i \pi t}\right)=1$ and $\mu_{C Z}\left(t \mapsto e^{-i \pi t}\right)=1$, respectively, where $t \in[0,1]$.
} 
manifold, the work of Abbondandolo and Majer [5] establishes existence of the Morse complex $\left(\mathrm{CM}_{*}\left(\mathcal{S}_{L}\right), \partial_{*}\left(\mathcal{S}_{L}, \mathcal{G}\right)\right)$ and shows that its homology is naturally isomorphic to the singular homology of the free loop space.

Given $L$ and $\mathcal{G}$ as in Step 2, the crucial third step is to construct a grading preserving chain complex isomorphism

$$
\Theta_{*}:\left(\mathrm{CM}_{*}\left(\mathcal{S}_{L}\right), \partial_{*}\left(\mathcal{S}_{L}, \mathcal{G}\right)\right) \longrightarrow\left(\mathrm{CF}_{*}\left(H_{L}\right), \partial_{*}\left(H_{L}, J\right)\right),
$$

where the Hamiltonian $H_{L}$ arises from the Lagrangian $L$ via Legendre transformation $(t, q, p) \mapsto(t, q, v(t, q, p))$. More precisely, define

$$
H_{L}(t, q, p):=\max _{v \in T_{q} M}(\langle p, v\rangle-L(t, q, v)) .
$$

For each $(t, q, p)$ the maximum is achieved at a unique point $v(t, q, p)$ by condition (L1). The Legendre transformation provides the natural identification $(x, y) \mapsto x$ of the critical points of $\mathcal{A}_{H_{L}}$ and $\mathcal{S}_{L}$. Hence both chain groups coincide. To define a chain homomorphism, fix $q \in \mathcal{P}(L)$ and $z \in \mathcal{P}\left(H_{L}\right)$. By definition, the moduli space $\mathcal{M}^{+}(q, z)$ consists of half cylinders $w:[0, \infty) \times S^{1} \rightarrow T^{*} M$ solving Floer's equation

$$
\bar{\partial}_{J, L} w:=\partial_{s} w+J(w) \partial_{t} w-\nabla H_{L}(t, w)=0
$$

such that $w(s, \cdot)$ converges uniformly to $z$, for $s \rightarrow+\infty$. The boundary condition at the other end requires the loop $w(0, \cdot)$ to project to the unstable manifold of $q$. This means that $u:=\pi \circ w(0, \cdot) \in W^{u}(q)$ where $\pi: T^{*} M \rightarrow M$ is the bundle projection.

The moduli space $\mathcal{M}^{+}(q, z)$ determines a Fredholm problem, because the unstable manifolds $W^{u}(q)$ are finite-dimensional and the boundary conditions for the $\bar{\partial}$-equation are Lagrangian and nondegenerate, respectively. For generic $J$ this moduli space is a smooth manifold of dimension $\operatorname{ind}_{L}(q)-\operatorname{ind}_{L}(z)$. In the case of equal indices, it is a discrete set and $\Theta_{*}$ is defined by counting its elements. Of course, compactness of the moduli space needs to be established first. Here a crucial observation of Abbondandolo and Schwarz enters, namely the inequality

$$
\mathcal{A}_{H_{L}}(x, y) \leq \mathcal{S}_{L}(x)
$$

for every loop $(x, y): S^{1} \rightarrow T^{*} M$. On critical points, equality holds. To prove compactness, a uniform action bound is needed to start with, but this follows immediately from equation (8): if $w \in \mathcal{M}^{+}(q, z)$, then $\mathcal{A}_{H_{L}}(w(s, \cdot)) \leq \mathcal{S}_{L}(q)$ for all $s \geq 0$. Compactness now follows from the $C^{0}$ estimate discussed in the first step. A gluing argument proves that $\Theta_{*}$ is a chain map. Moreover, inequality (8) shows that $\mathcal{M}^{+}(q, z)=\emptyset$ whenever $\mathcal{S}_{L}(q) \leq \mathcal{A}_{H_{L}}(z)$, unless $q$ and $z$ correspond to the same critical point. In this case, the set $\mathcal{M}^{+}(q, z)$ consists of a single element, the constant solution $w(s, \cdot)=z(\cdot)$. The inequality

$$
d^{2} \mathcal{A}_{H_{L}}(z)(\cdot, \cdot) \leq d^{2} \mathcal{S}_{L}(\pi(z))(D \pi(z) \cdot, D \pi(z) \cdot),
$$


the differential version of inequality (8) at $z \in \mathcal{P}\left(H_{L}\right)$, is used to prove that at a constant solution $w(s, \cdot)=z(\cdot)$ the Morse-Smale condition is automatically true. Ordering the generators of the chain groups by increasing action shows that $\Theta_{k}$ is an upper triangular matrix with diagonal entries \pm 1 .

\section{Singular perturbation and adiabatic limit}

This section reviews the approach by Salamon and Weber [18] who established a natural isomorphism $\operatorname{HF}_{*}^{a}\left(T^{*} M, H_{V}, J_{g}\right) \rightarrow \operatorname{HM}_{*}^{a}\left(\mathcal{L} M, \mathcal{S}_{V}, L^{2}\right)$. The main idea is to introduce a real parameter $\varepsilon>0$ in Floer's equations by replacing $\left(J_{g}, G_{g}\right)$ by

$$
\left(J_{\varepsilon}, G_{\varepsilon}\right):=\left(J_{\varepsilon^{-1} g}, G_{\varepsilon^{-1} g}\right) .
$$

This will not change Floer homology by Floer's continuation principle. Let us identify $T^{*} M$ with $T M$ via the metric isomorphism. A map $(\tilde{u}, \tilde{v})$ : $\mathbb{R} \times S^{1} \rightarrow T M$ is a solution to the $\left(J_{\varepsilon}, G_{\varepsilon}\right)$-Floer equations (6) if and only if $u(s, t):=\tilde{u}\left(\varepsilon^{-1} s, t\right)$ and $v(s, t):=v\left(\varepsilon^{-1} s, t\right)$ satisfy

$$
\partial_{s} u-\nabla_{t} v-\nabla V_{t}(u)=0, \quad \nabla_{s} v+\varepsilon^{-2}\left(\partial_{t} u-v\right)=0 .
$$

Denote the space of solutions to equations (7) and (9) by $\mathcal{M}^{\varepsilon}\left(x^{-}, x^{+} ; V\right)$. We shall outline how to prove that there is a one-to-one correspondence between the solutions of equations (3) and (9) subject to boundary conditions (4) and (7), respectively, whenever the index difference is one. ${ }^{4}$ This then shows that both chain complexes are identical. A first hint that such a bijection between parabolic and $\varepsilon$-elliptic flow lines exists is provided by the energy identity

$$
\begin{aligned}
E^{\varepsilon}(u, v) & :=\frac{1}{2} \int_{-\infty}^{\infty} \int_{0}^{1}\left(\left|\partial_{s} u\right|^{2}+\left|\nabla_{t} v+\nabla V_{t}(u)\right|^{2}+\varepsilon^{2}\left|\nabla_{s} v\right|^{2}+\varepsilon^{-2}\left|\partial_{t} u-v\right|^{2}\right) \\
& =\mathcal{S}_{V}\left(x^{-}\right)-\mathcal{S}_{V}\left(x^{+}\right)
\end{aligned}
$$

for the solutions of (7) and (9). It shows that $\partial_{t} u-v$ converges to zero in $L^{2}$ as $\varepsilon \rightarrow 0$. But if $\partial_{t} u=v$, then the first part in equation (9) is equivalent to equation (3).

The next step would be to prove that $\mathcal{S}_{V}$ is Morse-Smale for generic $V$. Unfortunately, this remains an open problem. Instead, we introduce a more general class of perturbations for which Morse-Smale transversality can be achieved generically by standard methods. These perturbations take the

\footnotetext{
${ }^{4}$ The case of arbitrary index difference is closely related to Cohen's conjecture [22]. It is the missing link in proving that cylindrical Gromov-Witten invariants of the cotangent bundle represent string topology of the free loop space. A different proof relating the particular cases of the three-point invariant provided by the pair of pants product in Floer homology and the Chas-Sullivan loop product $[\mathbf{2 3}]$ is in preparation by Abbondandolo and Schwarz [24].
} 
form of smooth functions $\mathcal{V}: \mathcal{L} M \rightarrow \mathbb{R}$ satisfying a list of axioms (which contains the properties used at some point in the proof; see [18]). Assume for the moment that $M$ was embedded isometrically in some Euclidean space $\mathbb{R}^{N}$, fix a loop $x_{0}$ in $M$ and let $\rho: \mathbb{R} \rightarrow[0,1]$ be a smooth cutoff function. Then a typical example of an abstract perturbation is given by

$$
\mathcal{V}(x):=\rho\left(\left\|x-x_{0}\right\|_{L^{2}}^{2}\right) \int_{0}^{1} V_{t}(x(t)) d t
$$

With new functionals $\mathcal{S}_{\mathcal{V}}:=\mathcal{S}_{0}+\mathcal{V}$ and $\mathcal{A}_{\mathcal{V}}:=\mathcal{A}_{0}+\mathcal{V}$, equation (9) becomes

$$
\partial_{s} u-\nabla_{t} v-\operatorname{grad} \mathcal{V}(u)=0, \quad \nabla_{s} v+\varepsilon^{-2}\left(\partial_{t} u-v\right)=0
$$

and the limit equation is of the form

$$
\partial_{s} u-\nabla_{t} \partial_{t} u-\operatorname{grad} \mathcal{V}(u)=0 .
$$

Here the $L^{2}$ gradient $\operatorname{grad} \mathcal{V}(x) \in \Omega^{0}\left(S^{1}, x^{*} T M\right)$ at $x \in \mathcal{L} M$ is defined by

$$
\int_{0}^{1}\left\langle\operatorname{grad} \mathcal{V}(u), \partial_{s} u\right\rangle d t:=\frac{d}{d s} \mathcal{V}(u)
$$

for every smooth path $\mathbb{R} \rightarrow \mathcal{L} M: s \mapsto u(s, \cdot)$. Let $\mathcal{P}(\mathcal{V})$ be given by $x \in \mathcal{L} M$ such that $\nabla_{t} \partial_{t} x=-\operatorname{grad} \mathcal{V}(x)$. Define $\mathcal{M}^{\varepsilon}\left(x^{-}, x^{+} ; \mathcal{V}\right)$ and $\mathcal{M}^{0}\left(x^{-}, x^{+} ; \mathcal{V}\right)$ as before with equations (10) and (11) replacing (9) and (3), respectively.

Let $V_{t}$ be a potential such that $\mathcal{S}_{V}$ is a Morse function and denote

$$
\mathcal{V}(x):=\int_{0}^{1} V_{t}(x(t)) d t
$$

Observe that this choice reproduces $\mathcal{S}_{V}$ and $\mathcal{A}_{V}$, hence the geometric equations (3) and (6). Fix a regular value $a$ of $\mathcal{S}_{V}$ and choose a sequence of perturbations $\mathcal{V}_{i}: \mathcal{L} M \rightarrow \mathbb{R}$ converging to $\mathcal{V}$ in the $C^{\infty}$ topology and such that $\mathcal{S}_{\mathcal{V}_{i}}: \mathcal{L} M \rightarrow \mathbb{R}$ is Morse-Smale for every $i$. We may assume without loss of generality that the perturbations agree with $\mathcal{V}$ near the critical points and that $\mathcal{P}\left(\mathcal{V}_{i}\right)=\mathcal{P}(V)$ for all $i$. Assume that there is a sequence $\varepsilon_{i}>0$ converging to zero such that, for every $\varepsilon_{i}$ and every pair $x^{ \pm} \in \mathcal{P}^{a}(V)$ of index difference 1 , there is a ( $s$-shift equivariant) bijection

$$
\mathcal{T}^{\varepsilon_{i}}: \mathcal{M}^{0}\left(x^{-}, x^{+} ; \mathcal{V}_{i}\right) \longrightarrow \mathcal{M}^{\varepsilon_{i}}\left(x^{-}, x^{+} ; \mathcal{V}_{i}\right)
$$


The following diagram - in which arrows represent isomorphisms and notation is simplified-shows how this implies our goal (with $\mathbb{Z}_{2}$-coefficients)

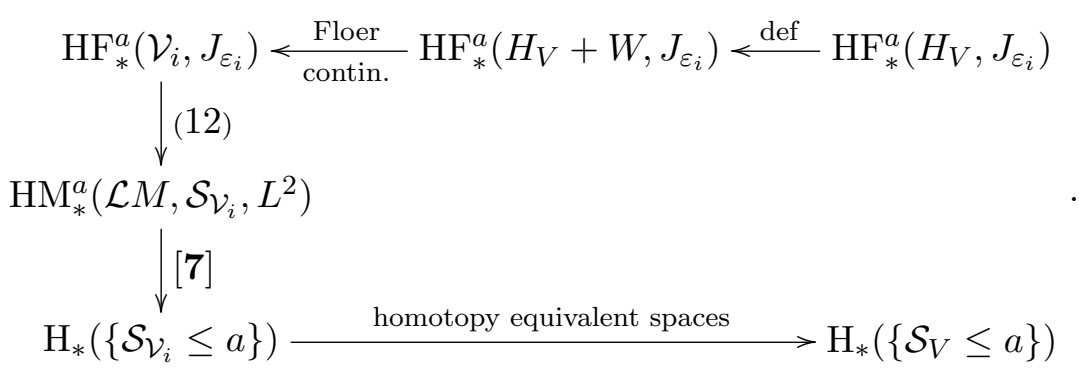

Starting at the upper right corner, the first step is by definition of Floer homology for nonregular $H_{V}$, namely add a small Hamiltonian perturbation $W$ making $H_{V}+W$ regular. Floer continuation shows independence of the choice. Again by Floer's continuation argument, small Hamiltonians and small abstract perturbations lead to isomorphic homology groups.

It remains to construct the bijection $\mathcal{T}^{\varepsilon}$ in the case of index difference 1 . Assume throughout that $\mathcal{S}_{\mathcal{V}}$ is Morse-Smale and fix a regular value $a$ and a pair $x^{ \pm} \in \mathcal{P}^{a}(V)$ of index difference 1. Denote $\mathcal{M}^{0}:=\mathcal{M}^{0}\left(x^{-}, x^{+} ; \mathcal{V}\right)$ and $\mathcal{M}^{\varepsilon}:=\mathcal{M}^{\varepsilon}\left(x^{-}, x^{+} ; \mathcal{V}\right)$.

Existence and uniqueness. Given a parabolic solution, we shall prove by Picard-Newton iteration existence and uniqueness of an elliptic solution nearby. The first step is to define, for smooth maps $(u, v): \mathbb{R} \times S^{1} \rightarrow T M$ and $p>2$, a map $\mathcal{F}_{u, v}^{\varepsilon}$ between the Banach spaces of $W^{1, p}$ and $L^{p}$ sections of the bundle $u^{*} T M \oplus u^{*} T M \rightarrow \mathbb{R} \times S^{1}$. To obtain uniform estimates for small $\varepsilon$, we introduce weighted norms. The weights for $p=2$ are suggested by the energy identity. Let $\mathcal{F}_{\varepsilon}(u, v)$ be given by the left-hand side of the $\varepsilon$-equations (9) and denote by $\Phi(x, \xi): T_{x} M \rightarrow T_{\exp _{x}(\xi)} M$ parallel transport along the geodesic $\tau \mapsto \exp _{x}(\tau \xi)$. For compactly supported vector fields $\zeta=(\xi, \eta) \in \Omega^{0}\left(\mathbb{R} \times S^{1}, u^{*} T M \oplus u^{*} T M\right)$ define the weighted norms

$$
\begin{aligned}
\|\zeta\|_{0, p, \varepsilon}:= & \left(\int_{-\infty}^{\infty} \int_{0}^{1}\left(|\xi|^{p}+\varepsilon^{p}|\eta|^{p}\right) d t d s\right)^{1 / p} \\
\|\zeta\|_{1, p, \varepsilon}:= & \left(\int _ { - \infty } ^ { \infty } \int _ { 0 } ^ { 1 } \left(|\xi|^{p}+\varepsilon^{p}|\eta|^{p}+\varepsilon^{p}\left|\nabla_{t} \xi\right|^{p}+\varepsilon^{2 p}\left|\nabla_{t} \eta\right|^{p}\right.\right. \\
& \left.\left.+\varepsilon^{2 p}\left|\nabla_{s} \xi\right|^{p}+\varepsilon^{3 p}\left|\nabla_{s} \eta\right|^{p}\right) d t d s\right)^{1 / p}
\end{aligned}
$$

and $\mathcal{F}_{u, v}^{\varepsilon}: W^{1, p}\left(\mathbb{R} \times S^{1}, u^{*} T M \oplus u^{*} T M\right) \rightarrow L^{p}\left(\mathbb{R} \times S^{1}, u^{*} T M \oplus u^{*} T M\right)$ by

$$
\mathcal{F}_{u, v}^{\varepsilon}\left(\begin{array}{c}
\xi \\
\eta
\end{array}\right):=\left(\begin{array}{cc}
\Phi(u, \xi)^{-1} & 0 \\
0 & \Phi(u, \xi)^{-1}
\end{array}\right) \mathcal{F}_{\varepsilon}\left(\begin{array}{c}
\exp _{u} \xi \\
\Phi(u, \xi)(v+\eta)
\end{array}\right) .
$$

Abbreviate $\mathcal{D}_{u, v}^{\varepsilon}:=d \mathcal{F}_{u, v}^{\varepsilon}(0,0)$ and $\mathcal{D}_{u}^{\varepsilon}:=\mathcal{D}_{u, \partial_{t} u}^{\varepsilon}$. 
Let us fix a parabolic cylinder $u \in \mathcal{M}^{0}$, viewed as an approximate solution of the $\varepsilon$-elliptic equations. Equivalently, we view the origin as approximate zero of the map $\mathcal{F}_{u}^{\varepsilon}:=\mathcal{F}_{u, \partial_{t} u}^{\varepsilon}$ between Banach spaces. Carrying out the Newton-Picard iteration for this map, we shall prove existence of a true zero nearby. The iteration method works if, firstly, the initial value is small, secondly, the linearized operator admits a right inverse, and thirdly, second derivatives of the map can be controlled. These conditions must be satisfied uniformly for small $\varepsilon>0$. Choosing the origin as the initial point of the iteration, we observe that

$$
\left\|\mathcal{F}_{u}^{\varepsilon}(0,0)\right\|_{0, p, \varepsilon}=\left\|\mathcal{F}_{\varepsilon}\left(u, \partial_{t} u\right)\right\|_{0, p, \varepsilon}=\left\|\left(0, \nabla_{s} \partial_{t} u\right)\right\|_{0, p, \varepsilon}=\varepsilon\left\|\nabla_{s} \partial_{t} u\right\|_{p} \leq c_{0} \varepsilon .
$$

The second identity uses the heat equation (3) and the final estimate is by exponential decay of heat flow solutions with nondegenerate boundary conditions. Verification of the second condition relies heavily on the fact that $\mathcal{D}_{u}^{\varepsilon}$ is Fredholm and surjective. (Fredholm follows by nondegeneracy of the boundary conditions (7) and surjectivity is a consequence of the MorseSmale assumption.) Let $\mathcal{D}_{u}^{\varepsilon *}$ be the adjoint operator of $\mathcal{D}_{u}^{\varepsilon}$ with respect to the $L^{2}$ inner product $\langle\cdot, \cdot\rangle_{\varepsilon}$ with associated norm $\|\cdot\|_{0,2, \varepsilon}$. A right inverse of $\mathcal{D}_{u}^{\varepsilon}$ is given by $\mathcal{Q}_{u}^{\varepsilon}:=\mathcal{D}_{u}^{\varepsilon *}\left(\mathcal{D}_{u}^{\varepsilon} \mathcal{D}_{u}^{\varepsilon *}\right)^{-1}$. It allows to solve the equation $0=\mathcal{D}_{u}^{\varepsilon} \zeta_{0}+\mathcal{F}_{u}^{\varepsilon}(0,0)$ and provides the correction term $\zeta_{0}:=-\mathcal{Q}_{u}^{\varepsilon} \mathcal{F}_{u}^{\varepsilon}(0,0)$. Recursively, for $\nu \in \mathbb{N}$, define the sequence of correction terms $\zeta_{\nu}$ by

$$
\zeta_{\nu}=\left(\xi_{\nu}, \eta_{\nu}\right):=-\mathcal{D}_{u}^{\varepsilon *}\left(\mathcal{D}_{u}^{\varepsilon} \mathcal{D}_{u}^{\varepsilon *}\right)^{-1} \mathcal{F}_{u}^{\varepsilon}\left(Z_{\nu}\right), \quad Z_{\nu}:=\sum_{\ell=0}^{\nu-1} \zeta_{\ell} .
$$

To estimate the terms $\zeta_{\nu}$, it is crucial to have an estimate for $\mathcal{D}_{u}^{\varepsilon}$ on the image of its adjoint operator. This is a consequence of a Calderon-Zygmund estimate for a Cauchy-Riemann-type operator. It follows that $Z_{\nu}$ is a Cauchy sequence. The third condition is verified by quadratic estimates for

$$
\mathcal{F}_{u}^{\varepsilon}\left(Z_{\nu}+\zeta_{\nu}\right)-\mathcal{F}_{u}^{\varepsilon}\left(Z_{\nu}\right)-d \mathcal{F}_{u}^{\varepsilon}\left(Z_{\nu}\right) \zeta_{\nu}, \quad d \mathcal{F}_{u}^{\varepsilon}\left(Z_{\nu}\right) \zeta_{\nu}-\mathcal{D}_{u}^{\varepsilon} \zeta_{\nu} .
$$

One proves by induction that there is a constant $c>0$ such that

$$
\left\|\mathcal{F}_{u}^{\varepsilon}\left(Z_{\nu+1}\right)\right\|_{0, p, \varepsilon^{3 / 2}} \leq \frac{c}{2^{\nu}} \varepsilon^{7 / 2-3 / 2 p}
$$

for all $\nu \in \mathbb{N}$ and $\varepsilon>0$ small. Hence the Cauchy sequence $Z_{\nu}$ converges to a zero $Z$ of $\mathcal{F}_{u}^{\varepsilon}$ with $Z \in \operatorname{im} \mathcal{D}_{u}^{\varepsilon *}$ and $\|Z\|_{1, p, \varepsilon} \leq c \varepsilon^{2}$. Now $Z=(X, Y)$ corresponds to a zero $\left(u^{\varepsilon}, v^{\varepsilon}\right)$ of $\mathcal{F}_{\varepsilon}$, in fact $\left(u^{\varepsilon}, v^{\varepsilon}\right) \in \mathcal{M}^{\varepsilon}$, and we define

$$
\mathcal{T}^{\varepsilon}(u):=\left(u^{\varepsilon}, v^{\varepsilon}\right):=\left(\exp _{u} X, \Phi(u, X)\left(\partial_{t} u+Y\right)\right) .
$$

Using again the quadratic estimates, one can prove uniqueness of the constructed solution $\left(u^{\varepsilon}, v^{\varepsilon}\right)$ in an even bigger neighborhood of $\left(u, \partial_{t} u\right)$. Here a crucial assumption is that the "difference" $Z$ between the parabolic and $\varepsilon$-elliptic solution is in the image of $\mathcal{D}_{u}^{\varepsilon *}$. It follows that $\mathcal{T}^{\varepsilon}$ is well defined. Moreover, it is time shift equivariant. Injectivity of $\mathcal{T}^{\varepsilon}$ follows, because 
the quotient $\mathcal{M}^{0} / \mathbb{R}$ by the time shift action is a finite set and so admits a positive smallest distance between its elements. Since the existence and uniqueness range shrinks like $\varepsilon^{2}$, injectivity holds for all sufficiently small $\varepsilon$.

Surjectivity. In four steps, we sketch the proof that the map $\mathcal{T}^{\varepsilon}: \mathcal{M}^{0} \rightarrow$ $\mathcal{M}^{\varepsilon}$ is surjective whenever $\varepsilon>0$ is sufficiently small.

The first step establishes uniform a priori $L^{\infty}$ bounds for elliptic solutions $\left(u^{\varepsilon}, v^{\varepsilon}\right) \in \mathcal{M}^{\varepsilon}$ and their first and second derivatives. In each case, this is based on first proving slicewise $L^{2}$ bounds, i.e., bounds in $L^{2}\left(S^{1}\right)$ for arbitrary fixed $s \in \mathbb{R}$, and then applying a mean value inequality for the operator $L_{\varepsilon}:=\varepsilon^{2} \partial_{s}^{2}+\partial_{t}^{2}-\partial_{s}$. More precisely, we prove that there is a constant $c>0$ such that for all $\varepsilon>0, r \in(0,1]$ and $\mu \geq 0$, the following is true. If $w$ is a smooth function (for instance, $w=\left|v^{\varepsilon}\right|^{2}$ ) defined on the parabolic domain $P_{r}^{\varepsilon}:=\left(-r^{2}-\varepsilon r, \varepsilon r\right) \times(-r, r)$, then

$$
L_{\varepsilon} w:=\left(\varepsilon^{2} \partial_{s}^{2}+\partial_{t}^{2}-\partial_{s}\right) w \geq-\mu w, \quad w \geq 0,
$$

imply

$$
w(0) \leq \frac{2 c e^{\mu r^{2}}}{r^{3}} \int_{P_{r}^{\varepsilon}} w .
$$

Now use the slicewise $L^{2}$ estimates on the right-hand side of the inequality to obtain the $L^{\infty}$ estimate. A bubbling argument enters the proof in the case of first derivatives, as is expected by comparison with standard Floer theory. It provides a rather weak (in terms of powers of $\varepsilon$ ) preliminary $L^{\infty}$ estimate which, however, suffices to establish the slicewise $L^{2}$ bounds.

Step 2 is to prove uniform exponential decay of $\left|\partial_{s} u^{\varepsilon}(s, t)\right|+\left|\nabla_{s} v^{\varepsilon}(s, t)\right|$ towards the ends of the cylinder. The standard method of proof works uniformly in $\varepsilon \in(0,1]$ under the assumption that the energy near the ends of the cylinder is uniformly bounded by a small constant (no energy concentration near infinity).

In Step 3, we establish local surjectivity by means of a time shift argument. Given $u \in \mathcal{M}^{0}$ and $\left(u^{\varepsilon}, v^{\varepsilon}\right) \in \mathcal{M}^{\varepsilon}$ sufficiently close to $\left(u, \partial_{t} u\right)$, define $\zeta=(\xi, \eta)$ by $u^{\varepsilon}=\exp _{u}(\xi)$ and $v^{\varepsilon}=\Phi(u, \xi)\left(\partial_{t} u+\eta\right)$. The idea is to prove that after a suitable time shift the pair $\zeta=(\xi, \eta)$ satisfies the crucial hypothesis $\zeta \in \operatorname{im} \mathcal{D}_{u}^{\varepsilon *}$ in the uniqueness theorem and therefore $\left(u^{\varepsilon}, v^{\varepsilon}\right)=\mathcal{T}^{\varepsilon}(u)$. More precisely, since the Fredholm index of $\mathcal{D}_{u}^{\varepsilon}$ is given by the Morse index difference and $\mathcal{D}_{u}^{\varepsilon}$ is surjective by the Morse-Smale assumption, it follows that $\operatorname{ker} \mathcal{D}_{u}^{\varepsilon}$ is generated by a nonzero vector $Z^{\varepsilon}$. Because $\operatorname{im} \mathcal{D}_{u}^{\varepsilon *}$ can be identified with the orthogonal complement of ker $\mathcal{D}_{u}^{\varepsilon}$ with respect to the $L^{2}$ inner product $\langle\cdot, \cdot\rangle_{\varepsilon}$, it remains to prove that the function

$$
\theta^{\varepsilon}(\sigma):=-\left\langle Z_{\sigma}^{\varepsilon}, \zeta_{\sigma}\right\rangle_{\varepsilon}, \quad Z^{\varepsilon}:=\left(\begin{array}{c}
\partial_{s} u \\
\nabla_{t} \partial_{s} u
\end{array}\right)-\mathcal{D}_{u}^{\varepsilon *}\left(\mathcal{D}_{u}^{\varepsilon} \mathcal{D}_{u}^{\varepsilon *}\right)^{-1} \mathcal{D}_{u}^{\varepsilon}\left(\begin{array}{c}
\partial_{s} u \\
\nabla_{t} \partial_{s} u
\end{array}\right)
$$

admits a zero. Here the $s$-shift is defined by $\zeta_{\sigma}(\cdot, \cdot):=\zeta(\cdot+\sigma, \cdot)$. 
In Step 4, we prove surjectivity. Assume by contradiction that there is a sequence $\left(u_{i}, v_{i}\right) \in \mathcal{M}^{\varepsilon_{i}}$ with $\varepsilon_{i}$ converging to zero and such that $\left(u_{i}, v_{i}\right) \notin$ $\mathcal{T}^{\varepsilon_{i}}\left(\mathcal{M}^{0}\right)$. Viewing the $u_{i}$ as approximate zeroes of the parabolic section $\mathcal{F}_{0}$, defined by the left-hand side of equation (3), we construct a sequence of parabolic solutions $u_{i}^{0} \in \mathcal{M}^{0}$ by Newton-Picard iteration. Here two conditions need to be satisfied uniformly for large $i \in \mathbb{N}$. Firstly, we need a small initial value of $\mathcal{F}_{0}$. This follows from the elliptic equations (9) and Step 1:

$$
\left\|\mathcal{F}_{0}\left(u_{i}\right)\right\|_{p}=\left\|\partial_{s} u_{i}-\nabla_{t} \partial_{t} u_{i}-\operatorname{grad} \mathcal{V}\left(u_{i}\right)\right\|_{p}=\varepsilon_{i}^{2}\left\|\nabla_{t} \nabla_{s} v_{i}\right\|_{p} \leq c \varepsilon_{i}^{2} .
$$

Secondly, one needs to prove asymptotic decay of the form

$$
\left|\partial_{s} u_{i}(s, t)\right|+\left|\nabla_{s} v_{i}(s, t)\right| \leq \frac{c}{1+s^{2}} .
$$

This follows from uniform exponential decay proved in Step 2. The iteration shows that $\left(u_{i}^{0}, \partial_{t} u_{i}^{0}\right)$ and the given elliptic solution $\left(u_{i}, v_{i}\right)$ are sufficiently close (for large $i$ ) such that the time shift argument of Step 3 applies. Hence $\left(u_{i}, v_{i}\right)=\mathcal{T}^{\varepsilon_{i}}\left(u_{i}^{0}\right)$, but this contradicts the assumption.

\section{Finite-dimensional approximation}

Reviewing Viterbo's paper [16] is somewhat delicate due to its state of presentation. As a way out, we decided to enlist the steps of proof as provided by [16] using the original notation and conventions. In view of its independent interest, we discuss Step 1 including full details (up to the hypothesis that both gradings coincide) in a separate section.

Let $(M, g)$ be a closed Riemannian manifold of dimension $n$. In [16], cohomology is considered and the main result is stated in the form

$$
\operatorname{HF}^{*}\left(D T^{*} M\right) \simeq \mathrm{H}^{*}(\mathcal{L} M),
$$

where $D T^{*} M$ denotes the open unit disc bundle. On both sides, contractible loops are considered and the isomorphism is claimed with rational coefficients. A further claim is a version of equation (13) for $S^{1}$-equivariant cohomologies (see [25] for applications). Since orientation of moduli space is not discussed in [16], we assume throughout Section 4 that all homologies take coefficients in $\mathbb{Z}_{2}$.

Actually [16] is part 2 of a series of two papers. The left-hand side of equation (13) is defined in part 1 [25] in the more general context of symplectic manifolds with contact type boundary (see the excellent recent survey by Oancea $[\mathbf{2 6}])$ : take the symplectic completion of the closed unit disc bundle (which is symplectomorphic to $\left(T^{*} M, \omega_{0}\right)$ itself), fix $\delta<1$ close to 1 and consider Hamiltonians of the form $H(t, q, p)=h_{\lambda}(|p|)$. Here $h_{\lambda}$ is a smooth convex real function which vanishes for $|p|<\delta$ and which is linear of slope $\lambda>0$ for $|p| \geq 1$. To define Floer cohomology associated to $h_{\lambda}$ it is important to assume that $\lambda$ is not the length of a contractible periodic 
geodesic in $M$ (see Remark 6.2). The left-hand side of equation (13) is then defined by the direct limit

$$
\operatorname{HF}^{*}\left(D T^{*} M\right):=\lim _{\lambda \rightarrow \infty} \operatorname{HF}^{*}\left(T^{*} M, h_{\lambda}, J_{g}\right) .
$$

To prove equation (13), it suffices to show $\operatorname{HF}^{*}\left(T^{*} M, h_{\lambda}, J_{g}\right) \simeq \mathrm{H}^{*}\left(\mathcal{L}^{\lambda^{2} / 2} M\right)$. The proof rests on the idea of Chaperon [27] to adapt the finite-dimensional approximation of the loop space via piecewise geodesics to the case of Hamiltonian flow lines. Givental [28] refined this idea for Hamiltonian flows which are periodic in time.

From now on fix $h=h_{\lambda}$, denote by $\varphi_{t}^{h}$ the time-t-map generated by the Hamiltonian vector field $X_{h}$, and set $\varphi^{h}:=\varphi_{1}^{h}$. The proof has seven steps:

$$
\begin{aligned}
\operatorname{HF}^{*}\left(T^{*} M, h, J_{g}\right) & \stackrel{1}{\simeq} \operatorname{HF}^{*}\left(\Delta_{r}, \Gamma_{r}\left(\varphi^{h}\right),\left(T^{*} M \times \overline{T^{*} M}\right)^{\times r}, J_{r}\right) \\
& \stackrel{2}{\simeq} \operatorname{HF}^{*}\left(U_{r, \varepsilon}, \operatorname{graph} d S_{\psi}, T^{*} U_{r, \varepsilon}, \Psi_{*} J_{r}\right) \\
& \stackrel{3}{\simeq} \operatorname{HM}^{*+r n}\left(U_{r, \varepsilon}, S_{\psi}\right) \\
& \stackrel{4}{\simeq} \mathrm{HI}^{*+r n}\left(U_{r, \varepsilon}, \nabla S_{\psi}\right) \\
& \stackrel{5}{\simeq} \mathrm{HI}^{*+r n}\left(U_{r, \varepsilon}, \xi_{\psi}\right) \\
& \stackrel{6}{\simeq} \mathrm{H}^{*}\left(\Lambda_{r, \varepsilon}^{a}\right) \\
& \stackrel{7}{\simeq} \mathrm{H}^{*}\left(\mathcal{L}^{\lambda^{2} / 2} M\right) .
\end{aligned}
$$

Here $\operatorname{HF}^{*}\left(L_{0}, L_{1} ; N\right)$ denotes Floer cohomology associated to Lagrangian submanifolds $L_{0}$ and $L_{1}$ of a symplectic manifold $N$, Morse cohomology is denoted by $\mathrm{HM}^{*}$ and the cohomological Conley index by HI*. The other symbols are introduced as they appear in our discussion. The whole proof relies on writing $\varphi^{h}$ as an $r$-fold composition $\psi^{r}:=\psi \circ \cdots \circ \psi$ for sufficiently large $r \in \mathbb{N}$. Since $h$ does not depend on time, we can choose $\psi:=\varphi_{1 / r}^{h}$.

Step 1. Let $\omega_{0}$ denote the canonical symplectic structure on $T^{*} M$, fix $r \in \mathbb{N}$ and consider the symplectic manifold

$$
\left(T^{*} M \times \overline{T^{*} M}\right)^{\times r}:=\left(\left(T^{*} M \times T^{*} M\right)^{\times r}, \omega_{r}:=\bigoplus_{1}^{r} \omega_{0} \oplus-\omega_{0}\right),
$$

and the Lagrangian submanifolds $\Delta_{r}:=\Delta^{\times r}$ and

$$
\Gamma_{r}\left(\varphi^{h}\right):=\left\{\left(z_{1}, \psi z_{r} ; z_{2}, \psi z_{1} ; \ldots ; z_{r}, \psi z_{r-1}\right) \mid z_{1}, \ldots, z_{r} \in T^{*} M\right\},
$$

where $\Delta$ denotes the diagonal in $T^{*} M \times T^{*} M$. Let $\mathcal{P}(h)$ denote the set of contractible 1-periodic orbits of $h$. Since

$$
\Gamma_{r}\left(\varphi^{h}\right) \cap \Delta_{r}=\left\{\left(z, z ; \psi z, \psi z ; \ldots ; \psi^{r-1} z, \psi^{r-1} z\right) \mid z \in \operatorname{Fix} \varphi^{h}\right\} \simeq \mathcal{P}(h),
$$


it follows that the generators of both chain groups coincide up to natural identification. That this identification preserves the grading given by the Conley-Zehnder index and the Maslov index, respectively, seems to be an open problem.

The boundary operator of Lagrangian intersection Floer homology counts $r$-tuples of pairs of $J_{r}$-holomorphic strips in $T^{*} M$, where $J_{r}$ is defined by equation (16). The boundary operator of periodic Floer homology counts $J_{g}$-holomorphic cylinders in $T^{*} M$. The idea to prove that both boundary operators coincide is to establish a one-to-one correspondence by gluing together the strips of an $r$-tuple to obtain a cylinder. Details of this argument will be discussed in a separate section below.

Remark 4.1 (Transversality). Working with time-independent Hamiltonians seems unrealistic at a first glance, since Morse-Smale transversality requires time dependence. We suggest perturbing $h$ in $C^{\infty}\left(\mathbb{R} / r^{-1} \mathbb{Z} \times\right.$ $\left.D T^{*} M\right)$. Due to the $1 / r$-periodicity of the perturbed Hamiltonian $H$, the crucial decomposition $\varphi^{H}=\psi^{r}$ is still available: fix an initial time, say $t_{0}=0$, and set $\psi:=\varphi_{0,1 / r}^{H}$. Since $H$ is still radial outside a compact set, Step 1 still goes through.

Step 2. In this step, coordinates are changed via a symplectomorphism which identifies $\Delta_{r}$ with $\left(T^{*} M\right)^{\times r}$ and the essential part of $\Gamma_{r}\left(\varphi^{h}\right)$ with the graph of the differential of a function. Let $d$ denote the Riemannian distance on $M$ and consider the neighborhood $\left(V_{\varepsilon}\right)^{\times r}$ of $\Delta_{r}$ where

$$
V_{\varepsilon}:=\left\{(q, p ; Q, P) \in T^{*} M \times T^{*} M \mid d(q, Q) \leq \varepsilon\right\} .
$$

Since $\Delta$ is diffeomorphic to $T^{*} M$, clearly $\Delta_{r}$ is diffeomorphic to $\left(T^{*} M\right)^{\times r}$. For sufficiently small $\varepsilon>0$, a proper symplectic embedding

$$
\Psi:\left(V_{\varepsilon}\right)^{\times r} \rightarrow T^{*}\left(T^{*} M\right)^{\times r}
$$

is constructed in $\left[\mathbf{2 0}\right.$, Lemma 1.1] identifying $\Delta_{r}$ with the zero section (see Figure 1). According to Viterbo [20], there exists $\varepsilon>0$ sufficiently small such that for every sufficiently large $r \in \mathbb{N}$ there is a function $S_{\psi}$ (denoted by $\mathcal{S}_{\Phi}$ in $[\mathbf{2 0}]$; note that $\psi$ depends on $r$ ) which is defined on the set

$$
U_{r, \varepsilon}:=\left\{\left(q_{1}, p_{1} ; \ldots ; q_{r}, p_{r}\right) \in\left(T^{*} M\right)^{\times r} \mid d\left(q_{j}, q_{j+1}\right) \leq \frac{\varepsilon}{2} \forall j \in \mathbb{Z}_{r}\right\} .
$$

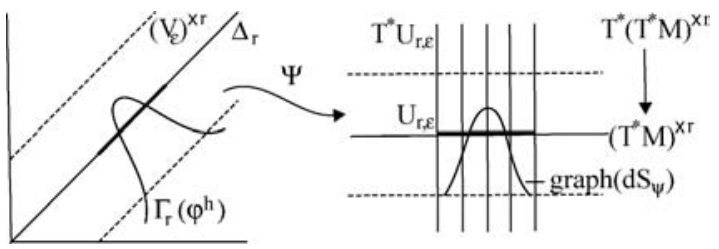

Figure 1. Change of coordinates $\Psi$. 
It has the property that the image under $\Psi$ of the part of $\Gamma_{r}\left(\varphi^{h}\right)$ contained in $\left(V_{\varepsilon}\right)^{\times r}$ equals the graph of $d S_{\psi}$.

To see existence of $S_{\psi}$, note that the Lagrangian $\Psi\left(\Gamma_{r}(\mathrm{id}) \cap\left(V_{\varepsilon}\right)^{\times r}\right)$ is a graph over $U_{r, \varepsilon}$ and $\Psi\left(\Gamma_{r}\left(\varphi^{h}\right) \cap\left(V_{\varepsilon}\right)^{\times r}\right)$ is close to this graph whenever $r$ is sufficiently large. Hence it is a Lagrangian graph over $U_{r, \varepsilon}$ itself. It is exact, because the symplectomorphism $\varphi^{h}$ is Hamiltonian. To arrive at the claim of Step 2, consider the two isomorphisms given by

$$
\begin{aligned}
& \operatorname{HF}^{*}\left(\Delta_{r}, \Gamma_{r}\left(\varphi^{h}\right),\left(T^{*} M \times \overline{T^{*} M}\right)^{\times r}, J_{r}\right) \\
& \quad \simeq \operatorname{HF}^{*}\left(\left(T^{*} M\right)^{\times r}, \Psi\left(\Gamma_{r}\left(\varphi^{h}\right) \cap\left(V_{\varepsilon}\right)^{\times r}\right), T^{*}\left(T^{*} M\right)^{\times r}, \Psi_{*} J_{r}\right) \\
& \quad \simeq \operatorname{HF}^{*}\left(U_{r, \varepsilon}, \operatorname{graph} d S_{\psi}, T^{*} U_{r, \varepsilon}, \Psi_{*} J_{r}\right) .
\end{aligned}
$$

The first isomorphism is induced by the symplectic embedding $\Psi$. One needs to prove that the Floer complex associated to $\left(\Delta_{r}, \Gamma_{r}\left(\varphi^{h}\right) ;\left(T^{*} M \times\right.\right.$ $\left.\overline{T^{*} M}\right)^{\times r} ; J_{r}$ ) lives entirely inside the neighborhood $\left(V_{\varepsilon}\right)^{\times r}$ of $\Delta_{r}$. This is clear for the generators, i.e., the points of intersection, but not so much for the connecting trajectories, i.e., the $J_{r}$-holomorphic strips. The second isomorphism follows, if again the whole Floer complex associated to the large space $T^{*}\left(T^{*} M\right)^{\times r}$ lives in the smaller space $T^{*} U_{r, \varepsilon}$. This is clear for the generators and for the connecting trajectories, a proof is given in [16].

Step 3. The Hamiltonian flow on $T^{*} U_{r, \varepsilon}$ generated by the Hamiltonian $(X, Y) \mapsto-S_{\psi}(X)$ is given by

$$
\phi_{t}(X, Y)=\left(X, Y+t d S_{\psi}(X)\right) .
$$

According to Viterbo [16], the Floer cohomologies associated to the almost complex structures $\Psi_{*} J_{r}$ and $\left(\phi_{t}\right)_{*} \Psi_{*} J_{r}$, respectively, are isomorphic by continuation. Furthermore, it is shown in [16] that the generators and connecting trajectories of the Floer complex associated to $\left(\phi_{t}\right)_{*} \Psi_{*} J_{r}$ are in one-to-one correspondence with those of the Morse complex associated to $\left(U_{r, \varepsilon}, \nabla S_{\psi}\right)$. Hence the corresponding cohomologies coincide up to a shift in the grading.

Step 4. Here HI* denotes the cohomological Conley index and the isomorphism is refered to $[\mathbf{2 9}]$.

Step 5. Existence of the pseudogradient vector field $\xi_{\psi}$ for $S_{\psi}$ is established in $[\mathbf{2 0}]$. Let $\mathrm{I}_{*}\left(U_{r, \varepsilon}, \xi_{\psi}\right)$ be the Conley index, i.e., the homotopy type of the quotient of $U_{r, \varepsilon}$ by the exit set with respect to the flow generated by $\xi_{\psi}$. According to $[\mathbf{1 6}]$, it holds

$$
\mathrm{I}_{*}\left(U_{r, \varepsilon}, \nabla S_{\psi}\right) \simeq \mathrm{I}_{*}\left(U_{r, \varepsilon / 2}, \xi_{\psi}\right),
$$

and the spaces are independent of $\varepsilon$ whenever $r$ is sufficiently large.

Step 6. Let $\varepsilon>0$ be smaller than the injectivity radius of $M$ and consider the finite-dimensional approximation of the free loop space given by

$$
\Lambda_{r, \varepsilon}:=\left\{\left(q_{1}, \ldots, q_{r}\right) \in M^{\times r} \mid d\left(q_{j}, q_{j+1}\right) \leq \frac{\varepsilon}{2} \forall j \in \mathbb{Z}_{r}\right\} .
$$


Define a function on $\Lambda_{r, \varepsilon}$ by

$$
E_{\psi}\left(q_{1}, \ldots, q_{r}\right):=\sup _{\left(p_{1}, \ldots, p_{r}\right)} S_{\psi}\left(q_{1}, p_{1} ; \ldots ; q_{r}, p_{r}\right),
$$

and set $\Lambda_{r, \varepsilon}^{a}:=\left\{E_{\psi} \leq a\right\}$. The Conley index of $\left(U_{r, \varepsilon}, \xi_{\psi}\right)$ is calculated in [20, Proposition. 1.7] and the result is the Thom space of some vector bundle of rank $r n$ over $\Lambda_{r, \varepsilon}^{a}$, for sufficiently large $a$. Taking cohomology, the Thom isomorphism leads to the shift in the grading and proves Step 6.

Step 7. According to Viterbo [20, p. 438], the space $\Lambda_{r, \varepsilon}^{a}$ approximates the free loop space $\mathcal{L}^{\lambda^{2} / 2} M$ for $a \rightarrow \infty, r \rightarrow \infty$ and $\varepsilon \rightarrow 0$.

Note that generating function homology has not been used throughout the seven steps - in contrast to what one expects after a glimpse into [16].

Step 1 revisited. We shall consider the case of Floer homology instead of Floer cohomology and continue our discussion of Step 1 above. First we review the original approach in $[\mathbf{1 6}]$. Then we propose a proof of the final argument along different lines.

To establish the isomorphism between periodic and Lagrangian Floer homology, it remains to check that the connecting trajectories arising in both situations are in one-to-one correspondence. In the case of periodic Floer homology, these are solutions $w: \mathbb{R} \times S^{1} \rightarrow T^{*} M$ satisfying

$$
\partial_{s} w+J_{g}(w) \partial_{t} w-\nabla h(w)=0,
$$

and appropriate boundary conditions. In the case of Lagrangian intersection Floer homology, these are $r$ pairs of strips $W:=\left(u_{1}, \hat{v}_{1} ; \ldots ; u_{r}, \hat{v}_{r}\right): \mathbb{R} \times$ $[0,1] \rightarrow\left(T^{*} M \times \overline{T^{*} M}\right)^{\times r}$ satisfying

$$
\partial_{s} W+J_{r}(W) \partial_{\tau} W=0, \quad W(s, 0) \in \Delta_{r}, \quad W(s, 1) \in \Gamma_{r}\left(\varphi^{h}\right) .
$$

Here we suggest to use the almost complex structure

$$
J_{r}=J_{r, \tau}:=\bigoplus_{1}^{r} J_{g} \oplus-\left(\varphi_{-\tau / r}^{h}\right)^{*} J_{g}
$$

in order to make the key idea in [16] work: achieve matching boundary conditions by redefining the $\hat{v}_{j}$ 's in the form $v_{j}(s, \tau):=\varphi_{-\tau / r}^{h} \circ \hat{v}_{j}(s, \tau)$. With this definition, equation (15) is equivalent to

$$
\begin{aligned}
\partial_{s} u_{j}+J_{g}\left(u_{j}\right) \partial_{\tau} u_{j} & =0, \quad v_{j}(s, 0)=u_{j}(s, 0), \\
\partial_{s} v_{j}-J_{g}\left(v_{j}\right) \partial_{\tau} v_{j} & =\frac{1}{r} \nabla h\left(v_{j}\right), \quad u_{j}(s, 1)=v_{j+1}(s, 1),
\end{aligned}
$$

for $j=1, \ldots, r$. Here and throughout, we identify $r+1$ and 1 . Note that the minus sign in the second PDE is fine, since we need to reverse time in the $v_{j}$ 's when fitting them together with the $u_{j}$ 's to obtain a perturbed $J$-holomorphic map $\tilde{w}: \mathbb{R} \times S^{1} \rightarrow T^{*} M$ (see Figure 2a). 
a)

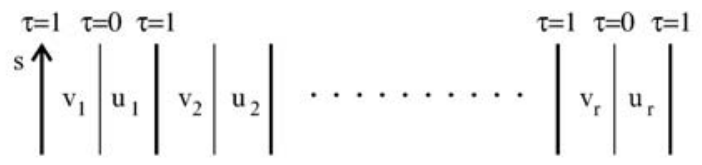

b)

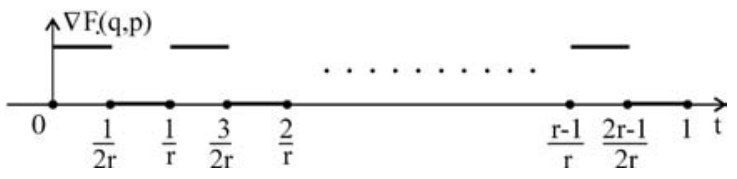

Figure 2. (a) Cylinder of $J$-holomorphic strips. (b) Time dependence of Hamiltonian perturbation $\nabla F$.

More precisely, define

$$
\tilde{w}(s, t):= \begin{cases}v_{j}(2 r s, 1-2 r t+2 j-2), & j \in\{1, \ldots, r\}, t \in\left[\frac{2 j-2}{2 r}, \frac{2 j-1}{2 r}\right], \\ u_{j}(2 r s, 2 r t-2 j+1), & j \in\{1, \ldots, r\}, t \in\left[\frac{2 j-1}{2 r}, \frac{2 j}{2 r}\right],\end{cases}
$$

and consider the perturbation associated to the Hamiltonian (see Figure 2b)

$$
F_{t}(q, p):= \begin{cases}2 h(|p|), & j \in\{1, \ldots, r\}, t \in\left[\frac{2 j-2}{2 r}, \frac{2 j-1}{2 r}\right] \\ 0, & \text { else. }\end{cases}
$$

The argument in $[\mathbf{1 6}]$ concludes as follows: the time-1-maps associated to $F$ and $h$ coincide, hence $\mathcal{P}(F) \simeq \mathcal{P}(h)$, and $\tilde{w}$ solves

$$
\partial_{s} \tilde{w}+J_{g}(\tilde{w}) \partial_{t} \tilde{w}-\nabla F_{t}(\tilde{w})=0,
$$

if the pairs $\left(u_{j}, v_{j}\right)$ solve equation (17). Hence both chain complexes are equal and

$$
\mathrm{HF}_{*}\left(\Delta_{r}, \Gamma_{r}\left(\varphi^{h}\right),\left(T^{*} M \times \overline{T^{*} M}\right)^{\times r}, J_{r}\right) \simeq \mathrm{HF}_{*}\left(T^{*} M, F, J_{g}\right) .
$$

According to Viterbo $[\mathbf{1 6}]$ continuation $^{5}$ then shows

$$
\mathrm{HF}_{*}\left(T^{*} M, F, J_{g}\right) \simeq \mathrm{HF}_{*}\left(T^{*} M, h, J_{g}\right) .
$$

\footnotetext{
${ }^{5}$ After writing this paper, Viterbo informed us that here continuation does not refer to Floer continuation but to the following: assume that the 1-periodic orbits do not depend on the parameter $\lambda \in[0,1]$ of a homotopy $f_{\lambda}$ between $F$ and $h$ and consider two 1-periodic orbits $z^{ \pm}$of index difference 1 . Set $X=\left\{(\lambda, w) \mid \partial_{s} w+J_{g}(w) \partial_{t} w=\right.$ $\left.\nabla f_{\lambda}(w), \lim _{s \rightarrow \pm \infty} w(s, t)=z^{ \pm}(t)\right\}$. Let $\mathbb{R}$ act on $X$ by $s$-shift of $w$. Then the projection $\pi: X / \mathbb{R} \rightarrow[0,1],(\lambda,[w]) \mapsto \lambda$, is Fredholm of index 0 . Hence the algebraic numbers of $\pi^{-1}(0)$ and $\pi^{-1}(1)$ are equal, provided we have compactness control. But these numbers represent the boundary matrix elements between $z^{-}$and $z^{+}$in the Floer complexes associated to $F$ and $h$, respectively. In the general case, as long as the 1-periodic orbits do not bifurcate, the same argument applies: the boundary conditions now depend on $\lambda$, but remain nondegenerate of constant index for all $\lambda$.
} 
The crucial point in defining the Floer homology associated to $F$ and constructing a Floer continuation homomorphism is to prove a priori $C^{0}$ estimates for the corresponding solutions. Both problems are nonstandard in the sense that $F$ as well as the homotopy of Hamiltonians are asymptotically nonconstant. Whereas a convexity argument can probably be adapted to solve the first problem, we do not see how to get the $C^{0}$ estimates needed for Floer continuation. Hence we propose a proof along different lines: in Remark 4.3 we switch on the Hamiltonian perturbation smoothly - simply to remain in the familiar setting of smooth Hamiltonians. Then, in Remark 4.4, we avoid asymptotically nonconstant Floer continuation altogether by introducing an intermediate step. All $C^{0}$ estimates we need will follow from [13, Proposition 2.3], which we recall below. Roughly speaking, it asserts that a Floer cylinder whose ends are located inside $D T^{*} M$ cannot leave this set, whenever outside the Hamiltonian is radial.

Proposition $4.2([\mathbf{1 3}])$. Given $R, c \geq 0$, let $f \in C^{\infty}\left(\mathbb{R} \times S^{1} \times[R, \infty)\right)$ satisfy $\partial_{s} f_{s, t}^{\prime} \geq 0$ and $f_{s, t}^{\prime \prime} \geq-c\left(\right.$ or $\left.f_{s, t}^{\prime \prime} \leq c\right)$ for all $s$ and $t$. Here $f_{s, t}(r):=f(s, t, r)$ and $f_{s, t}^{\prime}:=d / d r f_{s, t}$. Assume further that $H \in C^{\infty}\left(\mathbb{R} \times S^{1} \times T M\right)$ satisfies $H(s, t, x, y)=f_{s, t}(|y|)$ whenever $|y| \geq R$, that the pair $(u, v) \in C^{\infty}(\mathbb{R} \times$ $\left.S^{1}, T M\right)$ satisfies

$$
\left(\begin{array}{c}
\partial_{s} u-\nabla_{t} v \\
\nabla_{s} v+\partial_{t} u
\end{array}\right)-\nabla H(s, t, u, v)=0,
$$

and that there exists $T>0$ such that $|v(s, \cdot)| \leq R$ whenever $|s| \geq T$. Then $|v| \leq R$ on $\mathbb{R} \times S^{1}$.

Remark 4.3 (Smooth Hamiltonian). Smoothly switch off the Hamiltonian perturbation near the boundary of the $\hat{v}_{j}$-strips: fix a nondecreasing smooth $\operatorname{map} \alpha:[0,1] \rightarrow \mathbb{R}$ with $\alpha \equiv 0$ near 0 and $\alpha \equiv 1 / r$ near 1 (see Figure 3 ).

Define

$$
v_{j}(s, \tau):=\varphi_{-\alpha(\tau)}^{h} \circ \hat{v}_{j}(s, \tau), \quad J_{r}=J_{r, \tau}:=\bigoplus_{1}^{r} J_{g} \oplus-\left(\varphi_{-\alpha(\tau)}^{h}\right)^{*} J_{g} .
$$

Then the Lagrangian boundary value problem (15) is equivalent to

$$
\begin{aligned}
& \partial_{s} u_{j}+J_{g}\left(u_{j}\right) \partial_{\tau} u_{j}=0, \quad v_{j}(s, 0)=u_{j}(s, 0), \\
& \partial_{s} v_{j}-J_{g}\left(v_{j}\right) \partial_{\tau} v_{j}=\dot{\alpha}(\tau) \nabla h\left(v_{j}\right), \quad u_{j}(s, 1)=v_{j+1}(s, 1) \text {, }
\end{aligned}
$$
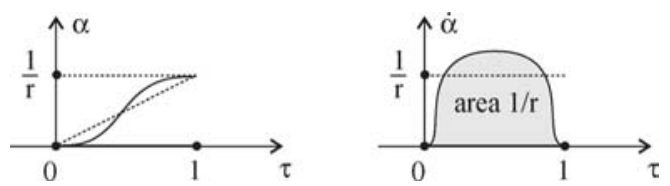

Figure 3. The function $\alpha$ and its derivative. 


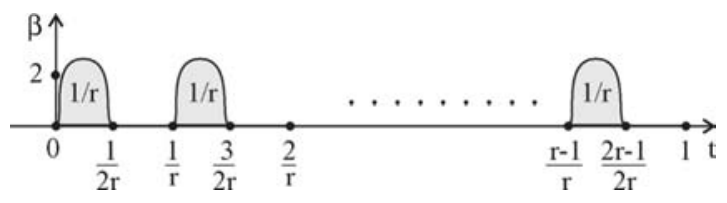

Figure 4. Time dependence of Hamiltonian perturbation $\beta \nabla h$.

for $j=1, \ldots, r$. Define a $1 / r$-periodic function (see Figure 4 ) by

$$
\beta(t):= \begin{cases}2 r \dot{\alpha}(1-2 r t+2 j-2), & j \in\{1, \ldots, r\}, t \in\left[\frac{2 j-2}{2 r}, \frac{2 j-1}{2 r}\right], \\ 0, & \text { else. }\end{cases}
$$

Consider the Hamiltonian $(t, q, p) \mapsto \beta(t) h(|p|)$, and let $\tilde{w}: \mathbb{R} \times S^{1} \rightarrow T^{*} M$ be given by equation (18). Then equation (19) is equivalent to

$$
\partial_{s} \tilde{w}+J_{g}(\tilde{w}) \partial_{t} \tilde{w}-\beta(t) \nabla h(\tilde{w})=0 .
$$

With these definitions, the Hamiltonian perturbations on the strips $\left(u_{j}, v_{j}\right)$ fit together smoothly, since $\nabla(\beta h)=\beta \nabla h$ depends smoothly on $t$ (see Figure 4). The fact that $\int_{0}^{1 / r} \beta(t) d t=1 / r$ and the identity $X_{\beta h}=\beta X_{h}$ together imply that the time- $1 / r$-maps associated to $\beta h$ and $h$, respectively, are equal. This proves $\operatorname{Fix} \varphi^{h}=\operatorname{Fix} \varphi^{\beta h}$ and $\mathcal{P}(h) \simeq \mathcal{P}(\beta h)$. The latter correspondence is given by mapping $z \in \mathcal{P}(h)$ to $\tilde{z}(\cdot):=z\left(\int_{0}^{\cdot} \beta(t) d t\right)$. Hence both chain complexes are equal and therefore

$$
\operatorname{HF}_{*}\left(\Delta_{r}, \Gamma_{r}\left(\varphi^{h}\right),\left(T^{*} M \times \overline{T^{*} M}\right)^{\times r}, J_{r} ; \mathbb{Z}_{2}\right) \simeq \operatorname{HF}_{*}\left(T^{*} M, \beta h, J_{g} ; \mathbb{Z}_{2}\right) .
$$

Again we remark that it is an open problem to prove equality of the gradings. Since the Hamiltonian $\beta h$ is not well behaved at infinity, definition of the right-hand side requires an additional argument to obtain a uniform $C^{0}$-bound for Floer trajectories: given our knowledge that all elements of $\mathcal{P}(\beta h)$ take values in $D T^{*} M$, the $C^{0}$ estimate for radial time-dependent Hamiltonians, Proposition 4.2 shows that all Floer cylinders connecting elements of $\mathcal{P}(\beta h)$ also take values in the bounded set $D T^{*} M$.

Remark 4.4 (Floer continuation). To conclude the proof of Step 1, we need to show that the Floer homologies of $\beta h$ and $h$, respectively, are isomorphic.

Choosing a different function $\beta$, if necessary, we may assume without loss of generality that $\beta_{m}:=\|\beta\|_{\infty}=3$. (Any real number strictly larger than 2 can be realized as such a maximum.) Pick a sufficiently large real $R_{0}>1$ and a smooth nondecreasing cutoff function $\rho$ which equals 0 on $(-\infty, 1]$ and 1 on $\left[R_{0}, \infty\right)$ such that the radial Hamiltonian

$$
h^{\rho, \beta(t)}(r):=(\rho(r)+\beta(t)(1-\rho(r))) h(r), \quad r=|p|
$$



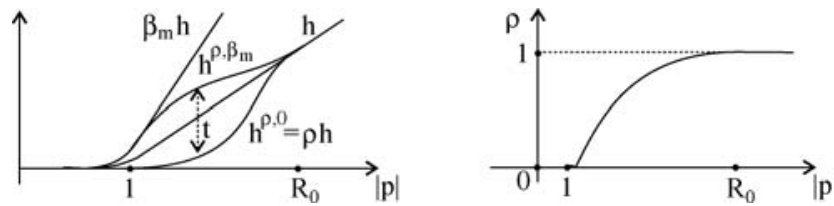

Figure 5. Family of Hamiltonians $h^{\rho, \beta(t)}$ and cutoff function $\rho$.

(see Figure 5) is nondecreasing for every $t \in[0,1]$. To check that such $R_{0}$ and $\rho$ exist is left as an exercise. (Hint: start with

$$
R_{0}:=-11 \frac{c_{\lambda}}{\lambda}+12, \quad \tilde{\rho}(r):= \begin{cases}0, & r \leq 1, \\ -\frac{\left(r-R_{0}\right)^{2}}{\left(1-R_{0}\right)^{2}}+1, & r \in\left[1, R_{0}\right], \\ 1, & r \geq R_{0},\end{cases}
$$

and check that indeed $\left(h^{\tilde{\rho}, \beta(t)}\right)^{\prime} \geq 0$ for every $t \in[0,1]$. Then smooth out $\tilde{\rho}$ near $r=1$ and $r=R_{0}$ using cutoff functions whose derivatives are supported in $[1,1+\mu]$ and $\left[R_{0}-\mu, R_{0}\right]$, respectively, for appropriate $\mu>0$.) In the $t$-variable, $h^{\rho, \beta(t)}$ oscillates between $h^{\rho, \beta_{m}}$ and $\rho h$ (see Figure 5). For $r \leq 1$, the Hamiltonian $h^{\rho, \beta}$ coincides with $\beta h$ and for $r \geq R_{0}$ with $h(r)=-c_{\lambda}+\lambda r$.

Since both $h$ and $h^{\rho, \beta}$ are linear of slope $\lambda$ outside $D_{R_{0}} T^{*} M$, any homotopy $f_{s}$ given by a convex combination of the two Hamiltonians satisfies $f_{s}^{\prime} \equiv \lambda$ outside $D_{R_{0}} T^{*} M$. Hence the two conditions $f_{s}^{\prime \prime} \equiv 0$ and $\partial_{s} f_{s}^{\prime} \equiv 0$ in Proposition 4.2 are satisfied for $R=R_{0}$ and the homotopy gives rise to a Floer continuation homomorphism. By the reverse homotopy argument

$$
\mathrm{HF}_{*}\left(T^{*} M, h, J_{g} ; \mathbb{Z}_{2}\right) \simeq \mathrm{HF}_{*}\left(T^{*} M, h^{\rho, \beta}, J_{g} ; \mathbb{Z}_{2}\right) .
$$

All elements of $\mathcal{P}(\beta h)$ and all connecting Floer trajectories associated to $\beta h$ take values in $D T^{*} M$ by the end of Remark 4.3. We claim that all elements of $\mathcal{P}\left(h^{\rho, \beta}\right)$ take values in $D T^{*} M$, too. Then by Proposition 4.2 the same is true for all Floer trajectories associated to $h^{\rho, \beta}$. But on $S^{1} \times D T^{*} M$ both Hamiltonians coincide, hence both chain complexes are equal and

$$
\mathrm{HF}_{*}\left(T^{*} M, h^{\rho, \beta}, J_{g} ; \mathbb{Z}_{2}\right) \simeq \mathrm{HF}_{*}\left(T^{*} M, \beta h, J_{g} ; \mathbb{Z}_{2}\right) .
$$

To prove the claim, consider the Hamiltonian vector field $X_{|p|^{2} / 2}$ generating the geodesic flow on $T^{*} M$. Since the Hamiltonian $h^{\rho, \beta}$ is radial, it holds

$$
X_{h^{\rho, \beta}}(q, p)=r^{-1}\left(h^{\rho, \beta}\right)^{\prime}(r) X_{r^{2} / 2}(q, p), \quad r=|p| .
$$

This shows that the projection to the zero section $M$ of a $X_{h^{\rho, \beta}}$-trajectory is a reparametrized geodesic. In particular, if $(x, y) \in \mathcal{P}\left(h^{\rho, \beta}\right)$, then $x: S^{1} \rightarrow M$ is a closed geodesic (not necessarily parametrized with respect to arc length) and $|y(t)|$ is independent of $t$. Assume by contradiction $r:=|y(t)| \geq 1$, then 
the length of the closed geodesic $x$ is given by

$$
\begin{aligned}
\ell(x) & =\int_{0}^{1}|\dot{x}(t)| d t=\int_{0}^{1}\left|\frac{\left(h^{\rho, \beta}\right)^{\prime}(r)}{r} g(x(t))^{-1} y(t)\right| d t \\
& =\int_{0}^{1}\left|\left(h^{\rho, \beta}\right)^{\prime}(r)\right| d t=\int_{0}^{1} \rho^{\prime}(1-\beta) h+(\rho+\beta(1-\rho)) \lambda d t \\
& =\rho^{\prime} h \int_{0}^{1}(1-\beta) d t+\rho \lambda \int_{0}^{1}(1-\beta) d t+\lambda \int_{0}^{1} \beta d t=\lambda .
\end{aligned}
$$

Here we used $\left(h^{\rho, \beta}\right)^{\prime} \geq 0$, the fact $h^{\prime}(r)=\lambda$ whenever $r \geq 1$ and $\int_{0}^{1} \beta=1$. But $\lambda$ was chosen in the complement of the length spectrum of $M$.

Remark 4.5 (closed aspherical case). Replacing the cotangent bundle by a closed symplectically aspherical manifold $(N, \omega)$, i.e., $\omega$ and the first Chern class vanish over $\pi_{2}(N)$, the argument of Step 1 in the case $r=1$ provides a new proof of the known fact

$$
\operatorname{HF}_{*}(N, H ; \mathbb{Z}) \simeq \operatorname{HF}_{*}\left(\Delta, \operatorname{graph} \varphi^{H} ; N \times \bar{N} ; \mathbb{Z}\right) .
$$

Note that Remark 4.4 can be replaced by taking right away a homotopy between $\beta H$ and $H$ and applying the standard reverse homotopy continuation argument. It is interesting to compare with the proof in $[\mathbf{3 0}]$.

\section{Example: the Euclidean torus}

We compute $L^{2}$ Morse homology of the loop space of the Euclidean torus $\mathbb{T}^{n}=\mathbb{R}^{n} / 2 \pi \mathbb{Z}^{n}$ and Floer homology of its cotangent bundle. Since the differential equations decouple, this essentially reduces to the case $n=1$.

$\boldsymbol{L}^{\mathbf{2}}$ Morse homology for $\mathcal{L}_{\boldsymbol{\alpha}} \mathbb{T}^{\mathbf{1}}$. Given $\alpha \in \mathbb{Z}$, we think of $x \in \mathcal{L}_{\alpha} \mathbb{T}^{1}$ as a smooth map $x: \mathbb{R} \rightarrow \mathbb{R}$ satisfying $x(t+1)=x(t)+2 \pi \alpha$. Hence $\alpha$ is the winding number of $x$. We fix $\alpha$ and analyze the problem for each component of the free loop space separately.

The equation for the free pendulum is $\partial_{t} \partial_{t} x=0$ and its solutions are given by $\left\{x(t)=2 \pi \alpha t+x_{0} \mid x_{0} \in \mathbb{T}^{1}\right\}$. Upon introducing a time-dependent potential on $S^{1} \times \mathbb{T}^{1}$ of the form $V_{\alpha}(t, q):=-\cos (q-2 \pi \alpha t)$, the circle of solutions splits into two nondegenerate critical points. Equation (1) for the critical points of the functional $\mathcal{S}_{V_{\alpha}}$ defined on $\mathcal{L}_{\alpha} \mathbb{T}^{1}$ becomes

$$
\partial_{t} \partial_{t} x=-\sin (x-2 \pi \alpha t) .
$$

Observe that the case $\alpha=0$ describes the mathematical pendulum with gravity. Obvious 1-periodic solutions of equation (20) are the two equilibrium states pendulum up and pendulum down. They are described by the constant functions $x^{(1)}=\pi$ and $x^{(0)}=0$, respectively. For general $\alpha$, equation (20) still describes the same mathematical pendulum, but now 
the observer rotates with constant angular speed $2 \pi \alpha$. The two equilibrium states of the pendulum are then seen by the observer as rotations and described by

$$
x^{(1)}=2 \pi \alpha t+\pi, \quad x^{(0)}=2 \pi \alpha t .
$$

Of course, the pendulum admits plenty of nonstationary periodic solutions, but their periods are strictly bigger than 1 . Hence $\mathcal{S}_{V_{\alpha}}$ has only the two critical points $x^{(1)}$ and $x^{(0)}$. Their actions are given by $2 \pi^{2} \alpha^{2}+1$ and $2 \pi^{2} \alpha^{2}-1$, respectively.

To compute the Morse index of $x^{(1)}$, we linearize equation $(20)$ at $x^{(1)}$ and solve the resulting eigenvalue problem $-\partial_{t} \partial_{t} \xi-\xi=\lambda \xi$ for $\xi: \mathbb{R} \rightarrow \mathbb{R}$ with $\xi(t+1)=\xi(t)$. The solution to this ODE is given by $\xi(t)=\sin \sqrt{\lambda+1} t$ and the periodicity condition implies $\lambda \in\left\{4 \pi^{2} k^{2}-1 \mid k=0,1,2, \ldots\right\}$. This shows $\operatorname{ind}_{V_{\alpha}}\left(x^{(1)}\right)=1$. Linearizing at $x^{(0)}$, we arrive at $-\partial_{t} \partial_{t} \xi+\xi=\lambda \xi$ with solution $\xi(t)=\sin \sqrt{\lambda-1} t$ and $\lambda \in\left\{4 \pi^{2} k^{2}+1 \mid k=0,1,2, \ldots\right\}$. Hence $\operatorname{ind}_{V_{\alpha}}\left(x^{(0)}\right)=0$. Therefore, the only nontrivial chain groups are given by

$$
C_{0}\left(V_{\alpha}\right)=\mathbb{Z} x^{(0)}, \quad C_{1}\left(V_{\alpha}\right)=\mathbb{Z} x^{(1)} .
$$

There must be precisely two connecting trajectories up to $s$-shift, since the unstable manifold of $x^{(1)}$ is one-dimensional. For $\sigma \in C^{\infty}(\mathbb{R}, \mathbb{R})$, consider the ansatz $u(s, t):=2 \pi \alpha t+\sigma(s)$. Then the heat equation (3) is equivalent to

$$
\sigma^{\prime}(s)=-\sin \sigma(s) \text {. }
$$

The initial conditions $\sigma(0)=0$ and $\sigma(0)=\pi$ produce the constant trajectories $u(s, t)=x^{(0)}(t)$ and $u(s, t)=x^{(1)}(t)$, respectively. The two 1-parameter families of connecting trajectories are obtained by choosing $\sigma(0)$ in $(0, \pi)$ or in $(-\pi, 0)$. Choosing an orientation of the unstable manifold of $x^{(1)}$ induces an orientation of each of the two 1-parameter families. For one family, this orientation coincides with the flow direction and for the other one it does not, so one characteristic sign is positive and one is negative. Hence $\partial^{M} x^{(1)}=x^{(0)}-x^{(0)}=0$. This proves that $L^{2}$ Morse homology of $\left(\mathcal{L}_{\alpha} \mathbb{T}^{1}, \mathcal{S}_{V_{\alpha}}\right)$ is in fact given by its chain groups $(22)$.

Floer homology of $\boldsymbol{T}^{*} \mathbb{T}^{\mathbf{1}}$. Let $V_{\alpha}$ be as above and define the Hamiltonian $H_{V_{\alpha}}(t, q, p):=1 / 2 p^{2}+V_{\alpha}(t, q)$ for $(t, q, p) \in S^{1} \times \mathbb{T}^{1} \times \mathbb{R}$. Hamilton's equations are given by

$$
\left(\begin{array}{l}
\dot{x}(t) \\
\dot{y}(t)
\end{array}\right)=\left(\begin{array}{c}
y(t) \\
-\sin (x(t)-2 \pi \alpha t)
\end{array}\right),
$$

and their two 1-periodic orbits by

$$
z^{(1)}(t)=\left(\begin{array}{c}
2 \pi \alpha t+\pi \\
2 \pi \alpha
\end{array}\right), \quad z^{(0)}(t)=\left(\begin{array}{c}
2 \pi \alpha t \\
2 \pi \alpha
\end{array}\right) .
$$

To calculate the two Floer trajectories, let $u$ be as above and set $v(s, t):=$ $2 \pi \alpha$. Floer's equations (3) are then equivalent to equation (23). The 
argument continues as above showing that $\mathrm{HF}_{*}\left(T^{*} \mathbb{T}^{1}, H_{V_{\alpha}}, J_{1} ; \alpha\right)$ equals the chain groups (22).

One might wonder what happens in the case of the $\varepsilon$-Floer equations (9). With our ansatz for $u$ and $v$, these are again equivalent to equation (23), in particular, $\varepsilon$ disappears. This means that the map $\mathcal{T}^{\varepsilon}: \mathcal{M}^{0} \rightarrow \mathcal{M}^{\varepsilon}$ is essentially the identity. It maps $u$ to $\left(u, \partial_{t} u\right)$ for any $\varepsilon>0$. The case $\varepsilon=1$ and our knowledge in the Morse case show that there are precisely two Floer trajectories up to $s$-shift.

Higher-dimensional case. Fix $n \geq 1$ and $\alpha=\left(\alpha_{1}, \ldots, \alpha_{n}\right) \in \mathbb{Z}^{n}$. On the Euclidean torus $\mathbb{T}^{n}=\mathbb{R}^{n} / \mathbb{Z}^{n}$, consider the potential $V_{\alpha}(t, q)$ given by the sum of $n$ one-dimensional potentials. The critical points of $\mathcal{S}_{V_{\alpha}}$ on $\mathcal{L}_{\alpha} \mathbb{T}^{n}$ are smooth functions $x: \mathbb{R} \rightarrow \mathbb{R}^{n}$ with $x(t+1)=x(t)+2 \pi \alpha$ satisfying

$$
\partial_{t} \partial_{t} x=-\nabla V_{\alpha}(t, x)=\left(-\sin \left(x_{1}-2 \pi \alpha_{1} t\right), \ldots,-\sin \left(x_{n}-2 \pi \alpha_{n} t\right)\right) .
$$

Its solutions are of the form $\left(x^{\left(\nu_{1}\right)}, \ldots, x^{\left(\nu_{n}\right)}\right)$ where the components $x^{\left(\nu_{k}\right)}$ are given by one of the two equilibrium states in (21). Hence the assignment $\left(x^{\left(\nu_{1}\right)}, \ldots, x^{\left(\nu_{n}\right)}\right) \mapsto\left(\nu_{1}, \ldots, \nu_{n}\right)=: \nu$ identifies the set of critical points with the direct sum $\left(\mathbb{Z}_{2}\right)^{n}$ of $n$ copies of $\mathbb{Z}_{2}$, and the number of critical points is $2^{n}$. Moreover, the Morse index of $x^{(\nu)}:=\left(x^{\left(\nu_{1}\right)}, \ldots, x^{\left(\nu_{n}\right)}\right)$ equals $|\nu|$ and

$$
C_{k}\left(V_{\alpha}\right)=\bigoplus_{\nu \in\left(\mathbb{Z}_{2}\right)^{n},|\nu|=k} x^{(\nu)} \mathbb{Z} \simeq \mathbb{Z}^{\left(\begin{array}{l}
n \\
k
\end{array}\right)} .
$$

Arguing as above, one observes that trajectories connecting critical points of index difference 1 come in pairs of opposite characteristic signs showing that all boundary operators are zero. (For example, in the case $n=3$ the critical point $(1,0,1)$ admits two connecting trajectories to $(0,0,1)$ and two to $(1,0,0))$. It follows that both $L^{2}$ Morse homology and Floer homology coincide with the chain groups (24) and, by Theorem 1.1, so does $\mathrm{H}_{*}\left(\mathcal{L}_{\alpha} \mathbb{T}^{n}\right)$.

\section{Application: noncontractible periodic orbits}

The search for noncontractible 1-periodic orbits is a fairly recent branch of symplectic geometry pioneered by Gatien-Lalonde [31] and BiranPolterovich-Salamon [30]. (See also [32] for some generalizations of $[\mathbf{3 1}]$.) These authors obtain existence results under certain geometric assumptions, for instance flatness of the metric. More precisely, Theorem 6.1 is proved in [30] for the Euclidean $n$-torus and in the case of negative curvature using the known simple structure of the set of periodic geodesics. In contrast, our extension to the case of arbitrary Riemannian metric is based on Theorem 1.1. 
Let $M$ be a closed connected Riemannian manifold. Given a homotopy class $\alpha$ of free loops in $M$, define the marked length spectrum $\Lambda_{\alpha}$ to be the set of lengths of all periodic geodesics representing $\alpha$. This set is closed and nowhere dense in $\mathbb{R}$. Hence $\ell_{\alpha}:=\inf \Lambda_{\alpha}$ belongs to $\Lambda_{\alpha}$. Let the open unit disc bundle $D T^{*} M$ be equipped with the canonical symplectic form $\omega_{0}$.

Theorem $6.1([\mathbf{1 3}])$. Let $\alpha$ be a homotopy class of free loops in $M$. Then every compactly supported Hamiltonian $H \in C^{\infty}\left([0,1] \times D T^{*} M\right)$ satisfying

$$
\sup _{[0,1] \times M} H=:-c \leq-\ell_{\alpha}
$$

admits a 1-periodic orbit $z=(x, y)$ with $[x]=\alpha$ and action $\mathcal{A}_{H}(z) \geq c$.

The idea of proof is to sandwich the Hamiltonian $H$ between two Hamiltonians $f$ and $h$ (see Figure 6) whose action filtered Floer homologies are computable and nonzero. Then prove that the monotonicity homomorphism associated to $f$ and $h$ is nonzero. This is a homomorphism from Floer homology of a given Hamiltonian to Floer homology of any pointwise larger Hamiltonian. Its crucial properties are, firstly, it respects the action window and, secondly, it factors through Floer homology of any third intermediate Hamiltonian. In our case, it factors through $\operatorname{HF}_{*}^{(a, \infty)}(H ; \alpha)$, which therefore is nonzero. Roughly speaking, if $c$ is a regular value of $\left.\mathcal{A}_{H}\right|_{\mathcal{L}_{\alpha} T^{*} M}$, set $a:=c$ and we are done.

The key tool to calculate the Floer homologies of $f$ and $h$ is a refinement of the Floer continuation principle. Namely, two Hamiltonians connected by an action regular homotopy (meaning that the boundary of the action window consists of regular values throughout the homotopy) have the same Floer homology. Action regularity is most easily checked for radial Hamiltonians.

Remark 6.2 (Radial Hamiltonians). Let $h: \mathbb{R} \rightarrow \mathbb{R}$ be a smooth symmetric function. A Hamiltonian of the form $H(t, q, p)=h(|p|)$ is called radial. Whenever the slope of $h$ at a point $r_{\ell}$ is equal to an element $\ell \in \Lambda_{\alpha}$, then

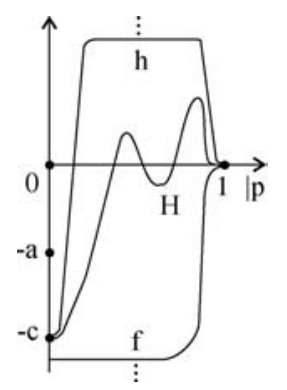

Figure 6. The Hamiltonian sandwich. 
there is a 1-periodic orbit $z_{\ell}$ of the Hamiltonian flow on the sphere bundle of radius $r_{\ell}$. All 1-periodic orbits arise that way. The symplectic action of $z_{\ell}$ equals minus the intercept of the tangent to the graph at the point $r_{\ell}$.

Hence we choose the Hamiltonians $f$ and $h$ radial. Then in each case we construct an action regular homotopy towards a convex radial Hamiltonian, since for these Floer homology is computed in [13] using Theorem 1.1. Of course, for different $H$ we may have to take new choices for $f$ and $h$. A convenient book-keeping tool to deal with this problem is to take the inverse limit over all $f$ and the direct limit over all $h$ subject to the restriction $\sup _{[0,1] \times M} h \leq-c$. These limits are called symplectic and relative symplectic homology and were introduced in $[\mathbf{3 3}, \mathbf{3 4}]$ and $[\mathbf{3 0}]$, respectively. As indicated in the diagram below, the monotone homomorphisms descend to a natural homomorphism $T$. The main part of $[\mathbf{1 3}]$ is devoted to establish and prove commutativity of the rectangular part of the following diagram:

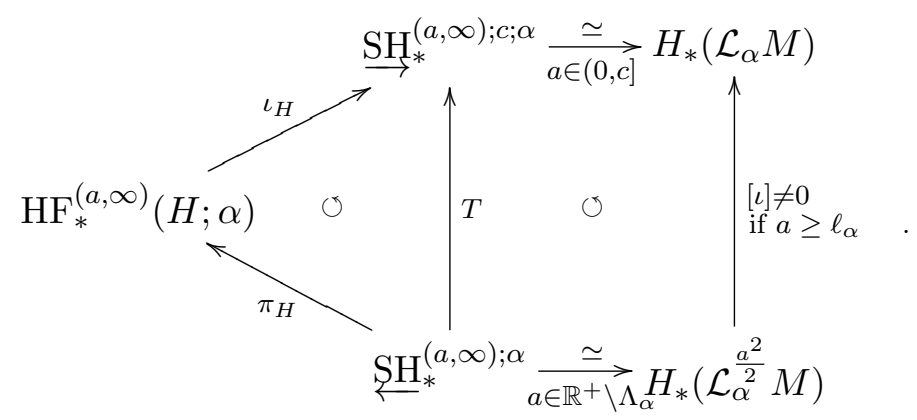

Note that the homomorphism $[\iota]$ induced by inclusion does not vanish whenever $a \geq \ell_{\alpha}$. It follows that $T$ is nonzero whenever $a \in\left[\ell_{\alpha}, c\right] \backslash \Lambda_{\alpha}$. In the case that $c$ is a regular value of $\left.\mathcal{A}_{H}\right|_{\mathcal{L}_{\alpha} T^{*} M}$ and $-c<-\ell_{\alpha}$ we can choose $a=c$ and are done. Otherwise choose a sequence of Hamiltonians $H_{\nu}$ converging to $H$ in $C^{\infty}$ and such that the corresponding $c_{\nu}$ satisfies both requirements above. From the resulting sequence of periodic orbits, extract a subsequence whose limit is the periodic orbit claimed by Theorem 6.1.

Consider the radial Hamiltonian whose graph in $\mathbb{R}^{2}$ consists of a straight line from $\left(0,-\ell_{\alpha}\right)$ to $(1,0)$ and which is zero elsewhere. Approximate it by a smooth function all of whose slopes are strictly less than $\ell_{\alpha}$ and which therefore does not admit any 1-periodic orbit representing $\alpha$. This example shows that the condition in Theorem 6.1 is sharp. It follows that the relative capacity defined in $[\mathbf{3 0}]$ and associated to $\left(D T^{*} M, M, \alpha\right)$ equals $\ell_{\alpha}$. As a byproduct, we obtain in $[\mathbf{1 3}]$ a multiplicity version of the Weinstein conjecture for compact hypersurfaces $Q \subset T^{*} M$ of contact type which enclose the zero section $M$. More precisely, for every nontrivial $\alpha$ we obtain existence of 
a closed characteristic on $Q$ whose projection to $M$ represents $\alpha$. In the nonsimply connected case, this refines the result of Hofer and Viterbo [35] in the case $\alpha=0$. Viterbo informed us that their techniques should also provide multiplicities.

\section{References}

[1] J. Milnor, Morse theory, Annals of Mathematics Studies 51, Princeton University Press, Princeton, NJ, 1964.

[2] E. Witten, Supersymmetry and Morse theory, J. Diff. Geom. 17 (1982), 661-692.

[3] M. Schwarz, Morse homology, PM 111, Birkhäuser, Basel, 1993.

[4] J. Weber, The Morse-Witten complex via dynamical systems, Expo. Math. 24 (2006), 127-159.

[5] A. Abbondandolo and P. Majer, Lectures on the Morse complex for infinite dimensional manifolds, Summer School on Morse theoretic methods in non-linear analysis and symplectic topology, Kluwert, Montreal, 2004.

[6] J. Weber, Perturbed closed geodesics are periodic orbits: index and transversality, Math. Z. 241 (2002), 45-81.

[7] J. Weber, The heat flow and the homology of the loop space, in preparation.

[8] A. Floer, Symplectic fixed points and holomorphic spheres, Commun. Math. Phys. 120 (1989), 575-611.

[9] D.A. Salamon, Lectures on Floer homology, In Symplectic Geometry and Topology, (Y. Eliashberg and L. Traynor, eds) IAS/Park City Mathematics Series, 7, 1999, $143-230$

[10] F. Laudenbach, Symplectic geometry and Floer homology, In Symplectic geometry and Floer homology. A survey of Floer homology for manifolds with contact type boundary or symplectic homology, Ensaios Mat. 7, Soc. Brasil. Mat., Rio de Janeiro, 2004, 1-50.

[11] D. McDuff and D.A. Salamon, J-holomorphic curves and Symplectic Topology, Colloquium Publications, 52, American Mathematical Society, Providence, RI, 2004.

[12] K. Cieliebak, Pseudo-holomorphic curves and periodic orbits on cotangent bundles, J. Math. Pures Appl. 73 (1994), 251-278.

[13] J. Weber, Noncontractible periodic orbits in cotangent bundles and Floer homology, Preprint, ETHZ, April 2004. arXiv:math.SG/0410609, Duke Math. J. 133 (2006), No. 3.

[14] A. Abbondandolo and M. Schwarz, On the Floer homology of cotangent bundles, Preprint, August 2004. arXiv:math.SG/0408280, Commun. Pure Appl. Math. 59 (2006), 254-316.

[15] C. Viterbo, Generating functions in symplectic topology and applications, In Proceedings ICM 94 Zürich, 1, Birkhäuser, Basel, 1995, 537-547.

[16] C. Viterbo, Functors and computations in Floer homology with applications, Part II, Preprint October 1996, revised 2003. http://math.polytechnique.fr/cmat/ viterbo/viterbo.html. 
[17] J. Weber, Morse theory on the loop space of flat tori and symplectic Floer theory, SFB 288 Geometrie \& Quantenphysik, Preprint 237, TU Berlin, 1996. arXiv:math.dg-ga/9612012.

[18] D.A. Salamon and J. Weber, Floer homology and the heat flow, Preprint, ETHZ, April 2003, revised September 2004. arXiv:math.SG/0304383, Geom. Funct. Anal., in press.

[19] J. Weber, J-holomorphic curves in cotangent bundles and the heat flow, Ph.D. thesis, TU Berlin, 1999.

[20] C. Viterbo, Exact Lagrange submanifolds, periodic orbits and the cohomology of free loop spaces, J. Diff. Geom. 47 (1997), 420-468.

[21] K. Cieliebak, Handle attaching in symplectic homology and the chord conjecture, J. Eur. Math. Soc. 4 (2002), 115-142.

[22] R. Cohen, Lectures on Morse theory, graphs, and string topology, Summer School on Morse theoretic methods in non-linear analysis and symplectic topology, Kluwert, Montreal, 2004. arXiv:math.GT/0411272.

[23] M. Chas and D. Sullivan, String topology, Preprint 1999. arXiv:math.GT/9911159.

[24] A. Abbondandolo and M. Schwarz, Floer homology of cotangent bundles, Summer School on Morse theoretic methods in non-linear analysis and symplectic topology, Kluwert, Montreal, 2004.

[25] C. Viterbo, Functors and computations in Floer homology with applications, I, Geom. Funct. Anal. 9 (1999), 985-1033.

[26] A. Oancea, A survey of Floer homology for manifolds with contact type boundary or symplectic homology, In Symplectic geometry and Floer homology. A survey of Floer homology for manifolds with contact type boundary or symplectic homology, Ensaios Mat. 7, Soc. Brasil. Mat., Rio de Janeiro, 2004, 51-91.

[27] M. Chaperon, Une idée du type "géodésiques brisées" pour les systèmes hamiltoniens, C. R. Acad. Sci. Paris 298 (1984), 293-396.

[28] A.B. Givental, Periodic maps in symplectic topology, Funct. Anal. Appl. (English translation) $\mathbf{2 3}$ (1989), 287-300.

[29] A. Floer, Witten's complex and infinite dimensional Morse theory, J. Diff. Geom. 30 (1989), 207-221.

[30] P. Biran, L. Polterovich and D.A. Salamon, Propagation in Hamiltonian dynamics and relative symplectic homology, Duke Math. J. 119 (2003), 65-118.

[31] D. Gatien and F. Lalonde, Holomorphic cylinders with Lagrangian boundaries and Hamiltonian dynamics, Duke Math. J. 102 (2000), 485-511.

[32] Y.-J. Lee, Non-contractible periodic orbits, Gromov invariants, and Floer-theoretic torsions, Preprint 2003. math.SG/0308185.

[33] A. Floer and H. Hofer, Symplectic homology. I. Open sets in $\mathbb{C}^{n}$, Math. Z. 215 (1994), 37-88.

[34] K. Cieliebak, A. Floer and H. Hofer, Symplectic homology. II. A general construction, Math. Z. 218 (1995), 103-122.

[35] H. Hofer and C. Viterbo, The Weinstein conjecture in cotangent bundles and related results, Ann. Sci. Norm. Sup. Pisa, Serie IV, Fasc. III, 15 (1988), 411-445.

We gratefully acknowledge partial financial support by DFG SPP 1154 Globale Differentialgeometrie, Scuola Normale Superiore Pisa and École Polytechnique Paris. We are 
particularly indebted to Alberto Abbondandolo, Kai Cieliebak, Pietro Majer, Dietmar Salamon, Matthias Schwarz, and Claude Viterbo for numerous helpful and pleasant conversations.

MAThematisches Institut

UNIVERSITÄT MÜNCHEN

D-80333 MÜNChEN GERMANY

E-mail address: joa@math.sunysb.edu

Received 03/01/2005, accepted 02/09/2006. 
